\title{
A Spectroscopic and Quantum Chemical Study of the Novel Compound Cyclopropylmethylselenol
}

\section{Supporting Information}

\section{George C. Cole, ${ }^{\dagger}{\text { Harald Møllendal, }{ }^{*}{ }^{\dagger} \text { and Jean-Claude Guillemin }}^{\ddagger}$}

Department of Chemistry, University of Oslo, Post Office Box 1033 Blindern, NO-0315 Oslo, Norway and Laboratoire de Synthèse et Activation de Biomolécules, Unité Mixte de Recherche 6052 du Centre National de la Recherche Scientifique, Institut de Chimie de Rennes, Ecole Nationale Supérieure de Chimie de Rennes, F-35700 Rennes, France

Corresponding author: e-mail harald.mollendal@kjemi.uio.no; tel. +47 2285 5458; fax +47 2285 5441

${ }^{\dagger}$ University of Oslo

${ }^{\ddagger}$ Ecole Nationale Supérieure de Chimie de Rennes 
Table 1S: Cyclopropylmethylselenol Conformer $\mathrm{V}\left({ }^{76} \mathrm{Se}\right)$. Ground vibrational state.

TOTAL NUMBER OF ACCEPTED TRANSITIONS: 26

\begin{tabular}{rrrrrr}
\multicolumn{7}{c}{ TRANSITION } \\
19 & 4 & 15 & 20 & 4 & 16 \\
19 & 4 & 16 & 20 & 4 & 17 \\
19 & 13 & 6 & 20 & 13 & 7 \\
19 & 13 & 7 & 20 & 13 & 8 \\
19 & 14 & 5 & 20 & 14 & 6 \\
19 & 14 & 6 & 20 & 14 & 7 \\
19 & 15 & 4 & 20 & 15 & 5 \\
19 & 15 & 5 & 20 & 15 & 6 \\
20 & 4 & 16 & 21 & 4 & 17 \\
20 & 4 & 17 & 21 & 4 & 18 \\
20 & 13 & 7 & 21 & 13 & 8 \\
20 & 13 & 8 & 21 & 13 & 9 \\
20 & 14 & 6 & 21 & 14 & 7 \\
20 & 14 & 7 & 21 & 14 & 8 \\
20 & 15 & 5 & 21 & 15 & 6 \\
20 & 15 & 6 & 21 & 15 & 7 \\
20 & 16 & 4 & 21 & 16 & 5 \\
20 & 16 & 5 & 21 & 16 & 6 \\
20 & 17 & 3 & 21 & 17 & 4 \\
20 & 17 & 4 & 21 & 17 & 5 \\
21 & 4 & 17 & 22 & 4 & 18 \\
21 & 4 & 18 & 22 & 4 & 19 \\
21 & 12 & 9 & 22 & 12 & 10 \\
21 & 12 & 10 & 22 & 12 & 11 \\
21 & 13 & 8 & 22 & 13 & 9 \\
21 & 13 & 9 & 22 & 13 & 10
\end{tabular}

OBS. FREQ. O. - C.

DISTORTION CONTRIBUTION

TOTAL

$52211.290-0.045$

$-7.60$

FIXED

$\begin{array}{llll}52209.670 & -0.154 & -7.60 & -0.01\end{array}$

$52185.730-0.021$

$52185.730-0.021$

8.32

$-\odot .03$

$52188.000 \quad 0.004$

8.32

$-\odot .03$

$52188.000 \quad 0.004$

11.11

$-0.04$

$52190.550-0.005$

11.11

$-0.04$

$52190.550-0.005$

14.12

$-\odot .06$

$54826.290 \quad 0.279$

14.12

$-\odot .06$

$54823.920 \quad 0.038$

$-8.99$

$-0.03$

$54794.550 \quad 0.072$

$-8.98$

$-0.02$

$54794.550 \quad 0.072$

7.75

$-0.03$

$54796.720-0.056$

7.75

$-0.03$

$54796.720-0.056$

10.68

$-0.04$

10.68

$-0.04$

$54799.520 \quad 0.104$

13.84

$-0.06$

$54799.520 \quad 0.104$

13.84

$-0.06$

$54802.270-0.096$

17.21

$-0.07$

$54802.270-0.096$

17.21

$-0.07$

$54805.650 \quad 0.043$

20.80

$-0.09$

$54805.650 \quad 0.043$

20.80

$-0.09$

$57441.270-0.090$

$-10.53$

$-0.04$

$57438.360-0.049$

$-10.51$

$-0.03$

4.18

$-0.01$

$57401.290 \quad 0.089$

4.18

$-0.01$

$57403.000-0.124$

7.03

$-0.02$

$57403.000-0.124$

7.03

$-0.02$

RMS $=0.1007 \quad$ GRAMDET.$=\odot .43 E-\odot 6$
ROTATIONAL CONSTANTS (MHZ):

$\begin{array}{rrrr}11127.710700 & 1344.833071 & 1263.755751 \\ +- & 0.000000 & 0.045188 & 0.051232\end{array}$

QUARTIC DISTORTION CONSTANTS:

$\begin{array}{rrrrrr} & 0.28881920 & -2.60227564 & 29.16152100 & 0.02701800 & 1.20187600 \\ +- & 0.00779495 & 0.01320014 & 0.0000000 \odot & 0.00000000 & 0.0000000 \odot\end{array}$

SIGNIFICANT DIGITS AND CORRELATION MATRIX:
16.000
$-0.95715 .000$
$0.128 \quad 0.164$
$-0.8810 .918$
8.000
$0.094 \quad 9.000$ 


\section{DERIVED CONSTANTS:}

KAPPA $=-\odot .983561$

MOMENTS OF INERTIA AND DEFECT $\left(U^{*} A^{* *} 2\right)$ : $45.41626428375 .79314558 \quad 399.90247278-21.30693709$

+- $\quad 0.00000000 \quad 0.01262697 \quad 0.01621178$

STANDARD DISTORTION CONSTANTS (KHZ):

$\begin{array}{rrr}-107.392258 & -1.371421 & -0.939133 \\ -0.866302 & -1.155277 & 8.964850\end{array}$


Table 2S: Cyclopropylmethylselenol Conformer V $\left({ }^{77} \mathrm{Se}\right)$. Ground vibrational state.

TOTAL NUMBER OF ACCEPTED TRANSITIONS: 36

\begin{tabular}{|c|c|c|c|c|c|c|c|c|c|}
\hline & & & & & & & & | & J \\
\hline & & & & & & & & & \\
\hline 19 & 12 & 7 & 20 & 12 & 8 & 51954.130 & $\odot .254$ & $\begin{array}{r}5.18 \\
5.18 L\end{array}$ & $\begin{array}{l}\text { FIXED } \\
-0.02\end{array}$ \\
\hline 19 & 12 & 8 & 20 & 12 & 9 & 51954.130 & 0.254 & 5.18 & -0.02 \\
\hline 19 & 14 & 5 & 20 & 14 & 6 & 51957.530 & $-\odot .079$ & 10.05 & $-\odot .04$ \\
\hline 19 & 14 & 6 & 20 & 14 & 7 & 51957.530 & -0.079 & 10.05 & -0.04 \\
\hline 19 & 15 & 4 & 20 & 15 & 5 & 51959.890 & -0.029 & 12.76 & -0.06 \\
\hline 19 & 15 & 5 & 20 & 15 & 6 & 51959.890 & $-\odot .029$ & 12.76 & $-\odot .06$ \\
\hline 19 & 16 & 3 & 20 & 16 & 4 & 51962.390 & -0.100 & 15.67 & $-\odot .07$ \\
\hline 19 & 16 & 4 & 20 & 16 & 5 & 51962.390 & -0.100 & 15.67 & $-\odot . \odot 7$ \\
\hline 19 & 17 & 2 & 20 & 17 & 3 & 51965.250 & $-\odot .056$ & 18.76 & $-\odot . \odot 9$ \\
\hline 19 & 17 & 3 & 20 & 17 & 4 & 51965.250 & $-\odot .056$ & 18.76 & -0.09 \\
\hline 20 & 4 & 16 & 21 & 4 & 17 & 54581.980 & 0.408 & -8.13 & -0.03 \\
\hline 20 & 4 & 17 & 21 & 4 & 18 & 54579.600 & $-\odot .207$ & -8.12 & $-\odot . \odot 2$ \\
\hline 20 & 13 & 7 & 21 & 13 & 8 & 54552.770 & $-\odot .089$ & $7.0 \odot$ & $-\odot . \odot 2$ \\
\hline 20 & 13 & 8 & 21 & 13 & 9 & 54552.770 & $-\odot . \odot 89$ & $7.0 \odot$ & $-\odot .02$ \\
\hline 20 & 15 & 5 & 21 & 15 & 6 & 54557.170 & -0.145 & 12.51 & $-\odot .05$ \\
\hline 20 & 15 & 6 & 21 & 15 & 7 & 54557.170 & -0.145 & 12.51 & $-\odot .05$ \\
\hline 20 & 16 & 4 & 21 & 16 & 5 & 54559.900 & $-\odot .079$ & 15.56 & $-\odot .07$ \\
\hline 20 & 16 & 5 & 21 & 16 & 6 & 54559.900 & -0.079 & 15.56 & -0.07 \\
\hline 21 & 4 & 17 & 22 & 4 & 18 & 57184.960 & $\odot .118$ & -9.52 & $-\odot . \odot 4$ \\
\hline 21 & 4 & 18 & 22 & 4 & 19 & 57182.220 & -0.176 & -9.50 & -0.03 \\
\hline 21 & 14 & 7 & 22 & 14 & 8 & 57152.190 & $\odot .016$ & 9.14 & $-\odot .03$ \\
\hline 21 & 14 & 8 & 22 & 14 & 9 & 57152.190 & $\odot .016$ & 9.14 & $-\odot .03$ \\
\hline 21 & 16 & 5 & 22 & 16 & 6 & 57157.550 & $\odot .175$ & 15.32 & $-\odot . \odot 7$ \\
\hline 21 & 16 & 6 & 22 & 16 & 7 & 57157.550 & 0.175 & 15.32 & -0.07 \\
\hline 21 & 17 & 4 & 22 & 17 & 5 & 57160.670 & $\odot .262$ & 18.71 & $-\odot . \odot 8$ \\
\hline 21 & 17 & 5 & 22 & 17 & 6 & 57160.670 & 0.262 & 18.71 & $-\odot . \odot 8$ \\
\hline 22 & 4 & 18 & 23 & 4 & 19 & 59788.700 & -0.065 & -11.05 & -0.06 \\
\hline 22 & 4 & 19 & 23 & 4 & 20 & 59785.350 & $-\odot .074$ & -11.03 & $-\odot . \odot 4$ \\
\hline 22 & 13 & 9 & 23 & 13 & 10 & 59747.240 & $\odot .054$ & 5.57 & $-\odot .01$ \\
\hline 22 & 13 & 10 & 23 & 13 & 11 & 59747.240 & 0.054 & 5.57 & $-\odot .01$ \\
\hline 22 & 14 & 8 & 23 & 14 & 9 & 59749.270 & $-\odot .06 \odot$ & 8.48 & $-\odot .03$ \\
\hline 22 & 14 & 9 & 23 & 14 & 10 & 59749.270 & $-\odot .06 \odot$ & 8.48 & $-\odot .03$ \\
\hline 22 & 15 & 7 & 23 & 15 & 8 & 59751.730 & -0.108 & 11.60 & $-\odot .04$ \\
\hline 22 & 15 & 8 & 23 & 15 & 9 & 59751.730 & -0.108 & 11.60 & $-\odot .04$ \\
\hline 22 & 17 & 5 & 23 & 17 & 6 & 59757.790 & -0.019 & 18.49 & -0.08 \\
\hline 22 & 17 & 6 & 23 & 17 & 7 & 59757.790 & -0.019 & 18.49 & -0.08 \\
\hline
\end{tabular}

RMS $=0.1520 \quad$ GRAMDET.$=0.77 \mathrm{E}-06$

$\begin{array}{rrrr}\text { ROTATIONAL CONSTANTS (MHZ) : } \\ \text { 11126.61140๑ } & 1337.261772 & 1259.883858 \\ +- & 0.00000 \odot & 0.057895 & 0.062736\end{array}$

QUARTIC DISTORTION CONSTANTS:

$\begin{array}{rrrrrr} & 0.26108401 & -2.35287843 & 29.16152100 & 0.02701800 & 1.20187600 \\ +- & 0.00606344 & 0.01521473 & 0.00000000 & 0.0000000 \odot & 0.0000000 \odot\end{array}$


n

SIGNIFICANT DIGITS AND CORRELATION MATRIX:

15.000

$-0.98115 .000$

$0.299-0.113 \quad 7.000$

$-0.880 \quad 0.910-0.125 \quad 8.000$

DERIVED CONSTANTS:

$\mathrm{KAPPA}=-0.984315$

MOMENTS OF INERTIA AND DEFECT $\left(U^{*} A^{* *} 2\right)$ :

$45.42075137377 .92080846 \quad 401.13145877-22.21010106$

+- $\quad 0.00000000 \quad 0.01636154 \quad 0.01997423$

STANDARD DISTORTION CONSTANTS (KHZ):
$-108.278906$
$-1.260480$
$-\odot .828192$
$-1.254155$
$-1.044336$
8.576997 
Table 3S: Cyclopropylmethylselenol Conformer V $\left({ }^{78} \mathrm{Se}\right)$. Ground vibrational state.

TOTAL NUMBER OF ACCEPTED TRANSITIONS: 134

$\begin{array}{rrrrrr} & \text { TRANSITION } & \\ & & & & & \\ 9 & 0 & 9 & 10 & 0 & 10 \\ 9 & 1 & 8 & 10 & 1 & 9 \\ 9 & 1 & 9 & 10 & 1 & 10 \\ 9 & 2 & 7 & 10 & 2 & 8 \\ 9 & 2 & 8 & 10 & 2 & 9 \\ 10 & 0 & 10 & 11 & 0 & 11 \\ 10 & 1 & 9 & 11 & 1 & 10 \\ 10 & 1 & 10 & 11 & 1 & 11 \\ 10 & 2 & 8 & 11 & 2 & 9 \\ 10 & 2 & 9 & 11 & 2 & 10 \\ 10 & 3 & 7 & 11 & 3 & 8 \\ 10 & 3 & 8 & 11 & 3 & 9 \\ 11 & 0 & 11 & 12 & 0 & 12 \\ 11 & 1 & 11 & 12 & 1 & 12 \\ 11 & 2 & 9 & 12 & 2 & 10 \\ 11 & 2 & 10 & 12 & 2 & 11 \\ 11 & 3 & 8 & 12 & 3 & 9 \\ 11 & 3 & 9 & 12 & 3 & 10 \\ 12 & 0 & 12 & 13 & 0 & 13 \\ 12 & 1 & 11 & 13 & 1 & 12 \\ 12 & 1 & 12 & 13 & 1 & 13 \\ 12 & 2 & 10 & 13 & 2 & 11 \\ 12 & 2 & 11 & 13 & 2 & 12 \\ 12 & 3 & 9 & 13 & 3 & 10 \\ 12 & 3 & 10 & 13 & 3 & 11 \\ 13 & 1 & 13 & 14 & 1 & 14 \\ 13 & 2 & 11 & 14 & 2 & 12 \\ 15 & 5 & 10 & 16 & 5 & 11 \\ 15 & 5 & 11 & 16 & 5 & 12 \\ 15 & 12 & 3 & 16 & 12 & 4 \\ 15 & 12 & 4 & 16 & 12 & 5 \\ 15 & 13 & 2 & 16 & 13 & 3 \\ 15 & 13 & 3 & 16 & 13 & 4 \\ 15 & 14 & 1 & 16 & 14 & 2 \\ 15 & 14 & 2 & 16 & 14 & 3 \\ 17 & 2 & 15 & 18 & 2 & 16 \\ 17 & 3 & 14 & 18 & 3 & 15 \\ 17 & 3 & 15 & 18 & 3 & 16 \\ 17 & 12 & 5 & 18 & 12 & 6 \\ 17 & 12 & 6 & 18 & 12 & 7 \\ 17 & 13 & 4 & 18 & 13 & 5 \\ 17 & 13 & 5 & 18 & 13 & 6 \\ 17 & 15 & 2 & 18 & 15 & 3 \\ 17 & 15 & 3 & 18 & 15 & 4 \\ 17 & 16 & 1 & 18 & 16 & 2 \\ 17 & 16 & 2 & 18 & 16 & 3 \\ 17 & 17 & 0 & 18 & 17 & 1 \\ 17 & 17 & 1 & 18 & 17 & 2 \\ 18 & 2 & 17 & 19 & 2 & 18\end{array}$

OBS. FREQ . O. - C.

$25787.330 \quad 0.047$

$26222.160 \quad 0.186$

$25462.670 \quad 0.037$

$25921.430-0.333$

$25849.280 \quad 0.262$

$28349.210 \quad 0.054$

$28839.170-0.103$

$28004.650-0.071$

$28527.530 \quad 0.099$

$28430.910 \quad 0.091$

28460.730

$\odot .227$

28458.470

0.053

$30906.500 \quad 0.028$

$30545.620-0.076$

$31136.420-0.272$

$31012.010 \quad 0.273$

$31050.620 \quad 0.054$

$31047.410 \quad 0.086$

$33458.790-0.197$

$34069.380 \quad 0.128$

$33085.610 \quad 0.120$

$33749.580-0.123$

$33591.730 \quad 0.038$

$33641.450-0.003$

$33636.670 \quad 0.074$

$35624.280 \quad 0.234$

$36366.470-0.079$

$41389.110-0.104$

$41389.110-0.102$

$41388.550 \quad 0.087$

$41388.550 \quad 0.087$

41390.350

0.150

41390.350

0.150

41392.280

0.112

41392.280

$\odot .112$

$46871.050 \quad 0.002$

$46612.400-0.184$

$46587.770-0.003$

$46561.450 \quad 0.140$

$46561.450 \quad 0.140$

$46563.390 \quad 0.219$

$46563.390 \quad 0.219$

$46567.250-0.451$

$46567.250-0.451$

$46570.030-0.301$

$46570.030-0.301$

46573. $090-0.096$

46573.090 - 0.096

$49046.970 \quad 0.063$

\section{DISTORTION CONTRIBUTION}

TOTAL

FIXED

$-1.05$

$-1.17$

$-0.90$

$-0.93$

$-0.88$

$-1.40$

$-1.56$

$-1.22$

$-1.30$

$-1.22$

$-0.96$

$-0.96$

$-1.80$

$-1.60$

$-1.74$

$-1.63$

$-1.36$

$-1.35$

$-2.27$

$-2.57$

$-2.04$

$-2.27$

$-2.11$

$-1.83$

$-1.83$

$-2.56$

$-2.90$

$-2.46$

$-2.46$

7.33

7.33

9.38

9.38

11.60

11.60

$-6.55$

$-5.69$

$-5.63$

6.90

6.90

9.21

9.21

14.38

14.38

17.24

17.24

20.29

20.29

$-6.96$
0.05

$-0.11$

$\odot .15$

$-0.03$

0.02

$\odot .07$

$-0.14$

๑. 20

$-0.05$

$\odot .03$

0.11

0.25

$-0.07$

0.04

0.15

$-0.21$

๑. 32

0.05

$-0.01$

0.40

$-0.14$

0.01

0.01

$-0.03$

$-0.03$

$-0.04$

$-0.04$

$-0.05$

$-0.05$

$-0.43$

$-0.07$

$-0.01$

$-0.02$

$-0.02$

$-0.04$

$-0.04$

$-0.06$

$-0.06$

$-0.07$

$-0.07$

$-0.09$

$-0.09$

๑. 22
$-0.10$ 


\begin{tabular}{|c|c|c|c|c|c|c|c|c|c|}
\hline 18 & 3 & 15 & 19 & 3 & 16 & 49211.140 & -0.034 & -6.82 & -0.10 \\
\hline 18 & 3 & 16 & 19 & 3 & 17 & 49178.720 & -0.003 & -6.72 & -0.01 \\
\hline 18 & 11 & 7 & 19 & 11 & 8 & 49146.030 & $-\odot .049$ & 4.26 & $-\odot .01$ \\
\hline 18 & 11 & 8 & 19 & 11 & 9 & 49146.030 & -0.049 & 4.26 & -0.01 \\
\hline 18 & 13 & 5 & 19 & 13 & 6 & 49149.570 & 0.019 & 8.94 & -0.03 \\
\hline 18 & 13 & 6 & 19 & 13 & 7 & 49149.570 & $\odot .019$ & 8.94 & $-\odot .03$ \\
\hline 18 & 14 & 4 & 19 & 14 & 5 & 49151.770 & $\odot .005$ & 11.58 & $-\odot .04$ \\
\hline 18 & 14 & 5 & 19 & 14 & 6 & 49151.770 & 0.005 & 11.58 & -0.04 \\
\hline 18 & 16 & 2 & 19 & 16 & 3 & 49156.890 & -0.114 & 17.43 & $-\odot .07$ \\
\hline 18 & 16 & 3 & 19 & 16 & 4 & 49156.890 & -0.114 & 17.43 & -0.07 \\
\hline 18 & 17 & 1 & 19 & 17 & 2 & 49159.880 & -0.113 & 20.65 & $-\odot .09$ \\
\hline 18 & 17 & 2 & 19 & 17 & 3 & 49159.880 & -0.113 & 20.65 & $-\odot . \odot 9$ \\
\hline 18 & 18 & 0 & 19 & 18 & 1 & 49163.170 & -0.046 & 24.06 & -0.11 \\
\hline 18 & 18 & 1 & 19 & 18 & 2 & 49163.170 & -0.046 & 24.06 & -0.11 \\
\hline 19 & 2 & 17 & 20 & 2 & 18 & 52142.300 & 0.146 & -9.16 & $-\odot .67$ \\
\hline 19 & 2 & 18 & 20 & 2 & 19 & 51618.030 & $\odot .032$ & -8.14 & 0.28 \\
\hline 19 & 3 & 16 & 20 & 3 & 17 & 51811.700 & $\odot . \odot 89$ & -8.07 & -0.14 \\
\hline 19 & 3 & 17 & 20 & 3 & 18 & 51769.700 & -0.087 & -7.94 & $-\odot .02$ \\
\hline 19 & 4 & 15 & 20 & 4 & 16 & $51757.00 \odot$ & 0.213 & -7.22 & $-\odot .02$ \\
\hline 19 & 4 & 16 & 20 & 4 & 17 & 51755.200 & -0.392 & -7.22 & -0.01 \\
\hline 19 & 12 & 7 & 20 & 12 & 8 & 51733.900 & $-\odot .0 \odot 6$ & 5.99 & -0.02 \\
\hline 19 & 12 & 8 & 20 & 12 & 9 & 51733.900 & $-\odot .006$ & 5.99 & $-\odot .02$ \\
\hline 19 & 14 & 5 & 20 & 14 & 6 & 51738.140 & $\odot . \odot \odot \odot$ & 11.33 & $-\odot .04$ \\
\hline 19 & 14 & 6 & 20 & 14 & 7 & 51738.140 & $0.00 \odot$ & 11.33 & -0.04 \\
\hline 19 & 15 & 4 & 20 & 15 & 5 & 51740.660 & -0.063 & 14.31 & $-\odot .06$ \\
\hline 19 & 15 & 5 & 20 & 15 & 6 & 51740.660 & -0.063 & 14.31 & -0.06 \\
\hline 19 & 16 & 3 & 20 & 16 & 4 & 51743.550 & -0.034 & 17.49 & $-\odot .07$ \\
\hline 19 & 16 & 4 & 20 & 16 & 5 & 51743.550 & -0.034 & 17.49 & $-\odot .07$ \\
\hline 19 & 17 & 2 & 20 & 17 & 3 & 51746.710 & ๑.0०5 & 20.88 & $-\odot .09$ \\
\hline 19 & 17 & 3 & 20 & 17 & 4 & 51746.710 & $\odot .005$ & 20.88 & -0.09 \\
\hline 19 & 18 & 1 & 20 & 18 & 2 & 51750.250 & $\odot .175$ & 24.47 & -0.11 \\
\hline 19 & 18 & 2 & 20 & 18 & 3 & 51750.250 & 0.175 & 24.47 & -0.11 \\
\hline 20 & 2 & 18 & 21 & 2 & 19 & 54781.170 & 0.187 & -10.69 & -0.82 \\
\hline 20 & 2 & 19 & 21 & 2 & 20 & 54187.470 & -0.068 & -9.45 & 0.34 \\
\hline 20 & 3 & 17 & 21 & 3 & 18 & 54414.000 & -0.084 & -9.48 & -0.19 \\
\hline 20 & 3 & 18 & 21 & 3 & 19 & 54360.900 & 0.006 & -9.30 & -0.02 \\
\hline 20 & 4 & 16 & 21 & 4 & 17 & 54347.960 & -0.209 & -8.55 & $-\odot .03$ \\
\hline 20 & 4 & 17 & 21 & 4 & 18 & 54346.570 & 0.086 & -8.54 & $-\odot .02$ \\
\hline 20 & 6 & 14 & 21 & 6 & 15 & 54323.150 & -0.105 & -6.33 & 0.02 \\
\hline 20 & 6 & 15 & 21 & 6 & 16 & 54323.150 & -0.105 & -6.33 & $\odot .02$ \\
\hline 20 & 11 & 9 & 21 & 11 & 10 & 54318.550 & $\odot .0 \odot 9$ & 2.86 & \\
\hline 20 & 11 & 10 & 21 & 11 & 11 & 54318.550 & $\odot . \odot \odot 9$ & 2.86 & \\
\hline 20 & 12 & 8 & 21 & 12 & 9 & 54320.170 & $\odot .069$ & 5.34 & -0.01 \\
\hline 20 & 12 & 9 & 21 & 12 & 10 & 54320.170 & $\odot .069$ & 5.34 & -0.01 \\
\hline 20 & 13 & 7 & 21 & 13 & 8 & 54322.150 & $\odot .068$ & 8.04 & $-\odot . \odot 2$ \\
\hline 20 & 13 & 8 & 21 & 13 & 9 & 54322.150 & $\odot .068$ & 8.04 & $-\odot . \odot 2$ \\
\hline 20 & 14 & 6 & 21 & 14 & 7 & 54324.500 & 0.073 & 10.95 & -0.04 \\
\hline 20 & 14 & 7 & 21 & 14 & 8 & 54324.500 & $\odot .073$ & 10.95 & $-\odot .04$ \\
\hline 20 & 15 & 5 & 21 & 15 & 6 & 54327.230 & 0.132 & 14.08 & -0.05 \\
\hline 20 & 15 & 6 & 21 & 15 & 7 & 54327.230 & 0.132 & 14.08 & -0.05 \\
\hline 20 & 16 & 4 & 21 & 16 & 5 & 54330.090 & $\odot .023$ & 17.42 & $-\odot .07$ \\
\hline 20 & 16 & 5 & 21 & 16 & 6 & 54330.090 & $\odot .023$ & 17.42 & $-\odot .07$ \\
\hline 20 & 17 & 3 & 21 & 17 & 4 & 54333.140 & -0.176 & 20.98 & $-\odot .09$ \\
\hline 20 & 17 & 4 & 21 & 17 & 5 & 54333.140 & -0.176 & 20.98 & $-\odot . \odot 9$ \\
\hline 20 & 18 & 2 & 21 & 18 & 3 & 54336.890 & 0.059 & 24.75 & -0.10 \\
\hline 20 & 18 & 3 & 21 & 18 & 4 & 54336.890 & 0.059 & 24.75 & -0.10 \\
\hline 21 & 2 & 20 & 22 & 2 & 21 & 56755.320 & -0.140 & -10.88 & 0.42 \\
\hline
\end{tabular}




$\begin{array}{rrrrrr}21 & 4 & 17 & 22 & 4 & 18 \\ 21 & 4 & 18 & 22 & 4 & 19 \\ 21 & 13 & 8 & 22 & 13 & 9 \\ 21 & 13 & 9 & 22 & 13 & 10 \\ 21 & 15 & 6 & 22 & 15 & 7 \\ 21 & 15 & 7 & 22 & 15 & 8 \\ 21 & 16 & 5 & 22 & 16 & 6 \\ 21 & 16 & 6 & 22 & 16 & 7 \\ 21 & 18 & 3 & 22 & 18 & 4 \\ 21 & 18 & 4 & 22 & 18 & 5 \\ 21 & 19 & 2 & 22 & 19 & 3 \\ 21 & 19 & 3 & 22 & 19 & 4 \\ 22 & 13 & 9 & 23 & 13 & 10 \\ 22 & 13 & 10 & 23 & 13 & 11 \\ 22 & 14 & 8 & 23 & 14 & 9 \\ 22 & 14 & 9 & 23 & 14 & 10 \\ 22 & 15 & 7 & 23 & 15 & 8 \\ 22 & 15 & 8 & 23 & 15 & 9 \\ 22 & 16 & 6 & 23 & 16 & 7 \\ 22 & 16 & 7 & 23 & 16 & 8 \\ 22 & 17 & 5 & 23 & 17 & 6 \\ 22 & 17 & 6 & 23 & 17 & 7 \\ 22 & 18 & 4 & 23 & 18 & 5 \\ 22 & 18 & 5 & 23 & 18 & 6 \\ 22 & 19 & 3 & 23 & 19 & 4 \\ 22 & 19 & 4 & 23 & 19 & 5 \\ 22 & 20 & 2 & 23 & 20 & 3 \\ 22 & 20 & 3 & 23 & 20 & 4\end{array}$

\begin{tabular}{|c|c|}
\hline 56940.030 & $-\odot .095$ \\
\hline 56937.530 & -0.259 \\
\hline 56908.170 & -0.055 \\
\hline & \\
\hline $13.40 \odot$ & $\odot .024$ \\
\hline 913.400 & $\odot .024$ \\
\hline 6916.570 & \\
\hline 6916.570 & 0.121 \\
\hline 923.630 & 0.154 \\
\hline 923.630 & 0.154 \\
\hline 5927.110 & -0.294 \\
\hline 56927.110 & -0.294 \\
\hline 59494.320 & \\
\hline 59494.320 & 0.039 \\
\hline 9496.750 & $\odot .023$ \\
\hline 9496.750 & 0.023 \\
\hline 9499.590 & 0.038 \\
\hline 59499.590 & $\odot .038$ \\
\hline 59502.920 & $\odot .197$ \\
\hline 59502.920 & $\odot .197$ \\
\hline 9506.290 & $\odot . \odot 76$ \\
\hline 506.290 & 0.076 \\
\hline 59510.090 & $\odot . \odot 83$ \\
\hline 59510.090 & 0.083 \\
\hline 59514.020 & -0.069 \\
\hline 59514.020 & -0.069 \\
\hline 59518.490 & 0.042 \\
\hline 59518.49 & 0.04 \\
\hline
\end{tabular}

$-10.01$

$-10.00$

7.38

7.38

13.71

13.71

17.21

17.21

24.89

24.89

29.07

29.07

6.58

6.58

9.77

9.77

13.19

13.19

16.85

16.85

20.75

20.75

24.88

24.88

29.25

29.25

33.86

33.86
$-0.04$

$-0.03$

$-0.02$

$-0.02$

$-0.05$

$-0.05$

$-0.07$

$-0.07$

$-0.10$

$-0.10$

$-0.12$

$-0.12$

$-0.01$

$-0.01$

$-0.03$

$-0.03$

$-0.04$

$-0.04$

$-0.06$

$-0.06$

$-0.08$

$-0.08$

$-0.10$

$-0.10$

$-0.12$

$-0.12$

$-0.14$

$-0.14$

RMS $=0.1423 \quad$ GRAMDET.$=0.31 \mathrm{E}-\odot 3$

ROTATIONAL CONSTANTS (MHZ):
$+-$
11052.667800
3. 210240
1331.101580
1255.012640
$\odot .004586$
0.004317

QUARTIC DISTORTION CONSTANTS:
$+-$
$\odot .27652172$
$-2.57922525$
29.16152100
$\odot .02701800$
1.20187600
$0.00180494 \quad 0.00318339$
0.00000000
0.00000000
0.00000000

\section{SIGNIFICANT DIGITS AND CORRELATION MATRIX:}

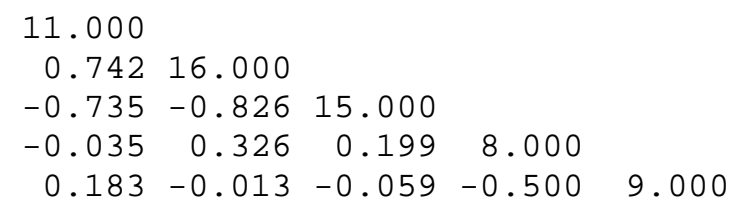

DERIVED CONSTANTS:

KAPPA $=-0.984468$

MOMENTS OF INERTIA AND DEFECT $(U * A * * 2)$ :

$$
\begin{array}{rrrrr} 
& 45.72462135 & 379.66978454 & 402.68841449 & -22.70599140 \\
+- & 0.01328069 & 0.00130797 & 0.00138508 & 0.01535612
\end{array}
$$


STANDARD DISTORTION CONSTANTS (KHZ):

$\begin{array}{rrr}-107.435270 & -1.322231 & -0.889943 \\ -0.863212 & -1.106087 & 8.967940\end{array}$


Table 4S: Cyclopropylmethylselenol Conformer V $\left({ }^{80} \mathrm{Se}\right)$. Ground vibrational state.

TOTAL NUMBER OF ACCEPTED TRANSITIONS: 116

\begin{tabular}{|c|c|c|c|c|c|c|c|c|c|}
\hline & & RAN & ITIO & & & OBS. FREO . & $0 .-C$. & DISTC & UTION \\
\hline & & & & & & & & TOTAL & FIXED \\
\hline 8 & $\odot$ & 8 & 9 & $\odot$ & 9 & 23029.840 & ๑. 012 & -0.75 & $\odot .03$ \\
\hline 8 & 1 & 7 & 9 & 1 & 8 & 23405.600 & $\odot .373$ & -0.83 & -0.09 \\
\hline 8 & 1 & 8 & 9 & 1 & 9 & 22732.410 & $\odot .219$ & -0.63 & $\odot .11$ \\
\hline 9 & $\odot$ & 9 & 10 & $\odot$ & 10 & 25575.140 & -0.033 & -1.03 & 0.05 \\
\hline 9 & 1 & 8 & 10 & 1 & 9 & 26002.300 & $\odot .328$ & -1.14 & -0.11 \\
\hline 9 & 1 & 9 & 10 & 1 & 10 & 25254.740 & 0.119 & -0.88 & 0.15 \\
\hline 9 & 2 & 7 & 10 & 2 & 8 & $257 \odot 4.960$ & $-\odot . \odot 49$ & -0.91 & -0.03 \\
\hline 9 & 2 & 8 & 10 & 2 & 9 & 25635.030 & ๑. 249 & -0.86 & 0.02 \\
\hline 9 & 3 & 6 & 10 & 3 & 7 & 25656.350 & $\odot .205$ & -0.62 & \\
\hline 9 & 3 & 7 & 10 & 3 & 8 & 25654.940 & 0.010 & -0.62 & \\
\hline 10 & 01 & 10 & 11 & $\odot$ & 11 & 28116.080 & -0.322 & -1.36 & $\odot .07$ \\
\hline 10 & 1 & 9 & 11 & 1 & 10 & 28597.130 & -0.314 & -1.52 & -0.14 \\
\hline 10 & 11 & 10 & 11 & 1 & 11 & 27776.020 & $-\odot .026$ & -1.18 & 0.20 \\
\hline 10 & 2 & 8 & 11 & 2 & 9 & 28288.180 & -0.366 & -1.26 & -0.05 \\
\hline 10 & 2 & 9 & 11 & 2 & 10 & 28195.480 & 0.216 & -1.18 & 0.03 \\
\hline 10 & 3 & 7 & 11 & 3 & 8 & 28224.140 & $\odot .24 \odot$ & -0.93 & \\
\hline 10 & 3 & 8 & 11 & 3 & 9 & 28221.970 & 0.044 & -0.93 & \\
\hline 11 & 11 & 10 & 12 & 1 & 11 & 31191.130 & $-\odot .359$ & -1.98 & $-\odot .18$ \\
\hline 11 & 11 & 11 & 12 & 1 & 12 & 30296.560 & $\odot .167$ & -1.55 & 0.25 \\
\hline 11 & 2 & 9 & 12 & 2 & 10 & 30875.530 & -0.034 & -1.69 & -0.07 \\
\hline 11 & 21 & 10 & 12 & 2 & 11 & 30755.080 & $\odot .187$ & -1.58 & 0.04 \\
\hline 11 & 3 & 8 & 12 & 3 & 9 & 30792.450 & $\odot .105$ & -1.31 & \\
\hline 11 & 3 & 9 & 12 & 3 & 10 & 30789.120 & -0.157 & -1.31 & \\
\hline 12 & 01 & 12 & 13 & $\odot$ & 13 & 33185.150 & -0.230 & -2.21 & 0.15 \\
\hline 12 & 11 & 11 & 13 & 1 & 12 & 33784.380 & $\odot .441$ & -2.52 & -0.21 \\
\hline 12 & 11 & 12 & 13 & 1 & 13 & $32815.80 \odot$ & 0.205 & -1.99 & 0.32 \\
\hline 12 & 21 & 10 & 13 & 2 & 11 & 33466.220 & -0.002 & -2.21 & -0.10 \\
\hline 12 & 21 & 11 & 13 & 2 & 12 & 33313.530 & $-\odot .062$ & -2.06 & 0.05 \\
\hline 12 & 3 & 9 & 13 & 3 & 10 & 33361.690 & 0.110 & -1.78 & -0.01 \\
\hline 12 & 31 & 10 & 13 & 3 & 11 & 33356.860 & -0.123 & -1.77 & \\
\hline 13 & 11 & 12 & 14 & 1 & 13 & 36374.690 & 0.069 & -3.14 & -0.25 \\
\hline 13 & 11 & 13 & 14 & 1 & 14 & 35333.420 & -0.178 & -2.49 & 0.39 \\
\hline 17 & 11 & 6 & 18 & 11 & 7 & 46173.550 & $\odot .242$ & 4.86 & -0.01 \\
\hline 17 & 11 & 7 & 18 & 11 & 8 & 46173.550 & 0.242 & 4.86 & -0.01 \\
\hline 17 & 13 & 4 & 18 & 13 & 5 & 46176.620 & -0.106 & 9.28 & -0.03 \\
\hline 17 & 13 & 5 & 18 & 13 & 6 & 46176.620 & -0.106 & 9.28 & -0.03 \\
\hline 17 & 14 & 3 & 18 & 14 & 4 & 46178.620 & -0.244 & 11.76 & -0.05 \\
\hline 17 & 14 & 4 & 18 & 14 & 5 & 46178.620 & -0.244 & 11.76 & -0.05 \\
\hline 17 & 15 & 2 & 18 & 15 & 3 & 46181.030 & $-\odot .222$ & 14.42 & -0.06 \\
\hline 17 & 15 & 3 & 18 & 15 & 4 & 46181.030 & -0.222 & 14.42 & $-\odot .06$ \\
\hline 17 & 16 & 1 & 18 & 16 & 2 & 46183.820 & -0.054 & 17.27 & -0.07 \\
\hline 17 & 16 & 2 & 18 & 16 & 3 & 46183.820 & -0.054 & 17.27 & -0.07 \\
\hline 17 & 17 & $\odot$ & 18 & 17 & 1 & 46186.750 & $\odot .030$ & 20.31 & -0.09 \\
\hline 17 & 17 & 1 & 18 & 17 & 2 & 46186.750 & 0.030 & 20.31 & -0.09 \\
\hline 18 & 11 & 7 & 19 & 11 & 8 & 48738.110 & $-\odot .047$ & 4.38 & -0.01 \\
\hline 18 & 11 & 8 & 19 & 11 & 9 & 48738.110 & $-\odot .047$ & 4.38 & -0.01 \\
\hline 18 & 12 & 6 & 19 & 12 & 7 & 48739.610 & -0.121 & 6.61 & -0.02 \\
\hline 18 & 12 & 7 & 19 & 12 & 8 & 48739.610 & -0.121 & 6.61 & -0.02 \\
\hline 181 & 13 & 5 & 19 & 13 & 6 & 48741.450 & -0.195 & 9.04 & -0.03 \\
\hline
\end{tabular}




\begin{tabular}{|c|c|c|c|c|c|c|c|c|c|}
\hline 18 & 13 & 6 & 19 & 13 & 7 & 48741.450 & -0.195 & $9 . \odot 4$ & -0.03 \\
\hline 18 & 14 & 4 & 19 & 14 & 5 & 48743.590 & -0.270 & 11.66 & -0.04 \\
\hline 18 & 14 & 5 & 19 & 14 & 6 & 48743.590 & -0.270 & 11.66 & -0.04 \\
\hline 18 & 15 & 3 & 19 & 15 & 4 & 48746.050 & -0.298 & 14.47 & -0.06 \\
\hline 18 & 15 & 4 & 19 & 15 & 5 & 48746.050 & -0.298 & 14.47 & -0.06 \\
\hline 18 & 16 & 2 & 19 & 16 & 3 & 48748.830 & -0.259 & 17.48 & $-\odot . \odot 7$ \\
\hline 18 & 16 & 3 & 19 & 16 & 4 & 48748.830 & -0.259 & 17.48 & $-\odot .07$ \\
\hline 19 & 3 & 16 & 20 & 3 & 17 & 51378.880 & 0.166 & -7.86 & -0.13 \\
\hline 19 & 3 & 17 & 20 & 3 & 18 & 51338.940 & -0.160 & -7.73 & $-\odot .02$ \\
\hline 19 & 4 & 15 & 20 & 4 & 16 & 51326.520 & 0.319 & -7.02 & $-\odot .02$ \\
\hline 19 & 4 & 16 & 20 & 4 & 17 & 51324.750 & -0.341 & -7.01 & -0.01 \\
\hline 19 & 12 & 7 & 20 & 12 & 8 & 51304.670 & 0.137 & 6.12 & -0.02 \\
\hline 19 & 12 & 8 & 20 & 12 & 9 & 51304.670 & 0.137 & 6.12 & $-\odot .02$ \\
\hline 19 & 13 & 6 & 20 & 13 & 7 & 51306.580 & $\odot .089$ & 8.68 & $-\odot .03$ \\
\hline 19 & 13 & 7 & 20 & 13 & 8 & 51306.580 & ๑.०89 & 8.68 & $-\odot .03$ \\
\hline 19 & 14 & 5 & 20 & 14 & 6 & 51308.950 & 0.174 & 11.43 & -0.04 \\
\hline 19 & 14 & 6 & 20 & 14 & 7 & 51308.950 & 0.174 & 11.43 & $-\odot .04$ \\
\hline 19 & 15 & 4 & 20 & 15 & 5 & 51311.500 & 0.142 & 14.40 & -0.06 \\
\hline 19 & 15 & 5 & 20 & 15 & 6 & 51311.500 & 0.142 & 14.40 & -0.06 \\
\hline 19 & 16 & 3 & 20 & 16 & 4 & 51314.290 & 0.077 & 17.56 & $-\odot .07$ \\
\hline 19 & 16 & 4 & 20 & 16 & 5 & 51314.290 & 0.077 & 17.56 & $-\odot .07$ \\
\hline 20 & 3 & 17 & 21 & 3 & 18 & 53959.020 & -0.010 & -9.23 & -0.18 \\
\hline 20 & 3 & 18 & 21 & 3 & 19 & 53908.570 & -0.073 & -9.06 & $-\odot .02$ \\
\hline 20 & 4 & 16 & 21 & 4 & 17 & 53896.020 & 0.110 & -8.31 & -0.03 \\
\hline 20 & 4 & 17 & 21 & 4 & 18 & 53894.290 & -0.056 & -8.30 & $-\odot .02$ \\
\hline 20 & 11 & 9 & 21 & 11 & 10 & 53867.820 & 0.133 & 3.04 & \\
\hline 20 & 11 & 10 & 21 & 11 & 11 & 53867.820 & 0.133 & 3.04 & \\
\hline 20 & 12 & 8 & 21 & 12 & 9 & 53869.320 & $\odot .054$ & 5.50 & -0.01 \\
\hline 20 & 12 & 9 & 21 & 12 & 10 & 53869.320 & $\odot .054$ & 5.50 & $-\odot .01$ \\
\hline 20 & 13 & 7 & 21 & 13 & 8 & 53871.470 & 0.212 & 8.19 & $-\odot .02$ \\
\hline 20 & 13 & 8 & 21 & 13 & 9 & 53871.470 & 0.212 & 8.19 & -0.02 \\
\hline 20 & 14 & 6 & 21 & 14 & 7 & 53873.670 & 0.063 & 11.08 & -0.04 \\
\hline 20 & 14 & 7 & 21 & 14 & 8 & 53873.670 & $\odot .063$ & 11.08 & $-\odot .04$ \\
\hline 20 & 15 & 5 & 21 & 15 & 6 & 53876.390 & 0.113 & 14.19 & -0.05 \\
\hline 20 & 15 & 6 & 21 & 15 & 7 & 53876.390 & 0.113 & 14.19 & -0.05 \\
\hline 20 & 16 & 4 & 21 & 16 & 5 & 53879.320 & $\odot .079$ & 17.52 & $-\odot .07$ \\
\hline 20 & 16 & 5 & 21 & 16 & 6 & 53879.320 & $\odot .079$ & 17.52 & $-\odot .07$ \\
\hline 20 & 17 & 3 & 21 & 17 & 4 & 53882.540 & $\odot .058$ & 21.05 & $-\odot .08$ \\
\hline 20 & 17 & 4 & 21 & 17 & 5 & 53882.540 & 0.058 & 21.05 & -0.08 \\
\hline 21 & 3 & 18 & 22 & 3 & 19 & 56541.660 & 0.181 & -10.75 & -0.24 \\
\hline 21 & 3 & 19 & 22 & 3 & 20 & 56477.910 & -0.258 & -10.51 & $-\odot .03$ \\
\hline 21 & 4 & 17 & 22 & 4 & 18 & 56466.160 & -0.009 & -9.74 & $-\odot .04$ \\
\hline 21 & 4 & 18 & 22 & 4 & 19 & 56464.070 & 0.070 & -9.73 & $-\odot .03$ \\
\hline 21 & 13 & 8 & 22 & 13 & 9 & 56435.920 & -0.024 & 7.56 & $-\odot .02$ \\
\hline 21 & 13 & 9 & 22 & 13 & 10 & 56435.920 & -0.024 & 7.56 & -0.02 \\
\hline 21 & 14 & 7 & 22 & 14 & 8 & 56438.270 & -0.079 & 10.59 & $-\odot .03$ \\
\hline 21 & 14 & 8 & 22 & 14 & 9 & 56438.270 & -0.079 & 10.59 & -0.03 \\
\hline 21 & 15 & 6 & 22 & 15 & 7 & 56441.140 & 0.038 & 13.85 & -0.05 \\
\hline 21 & 15 & 7 & 22 & 15 & 8 & 56441.140 & 0.038 & 13.85 & -0.05 \\
\hline 21 & 16 & 5 & 22 & 16 & 6 & 56444.340 & 0.170 & 17.33 & $-\odot .06$ \\
\hline 21 & 16 & 6 & 22 & 16 & 7 & 56444.340 & 0.170 & 17.33 & $-\odot .06$ \\
\hline 22 & 3 & 19 & 23 & 3 & 20 & 59126.130 & -0.123 & -12.43 & -0.32 \\
\hline 22 & 3 & 20 & 23 & 3 & 21 & 59047.480 & -0.114 & -12.11 & $-\odot .03$ \\
\hline 22 & 4 & 18 & 23 & 4 & 19 & 59036.820 & -0.200 & -11.32 & -0.06 \\
\hline 22 & 4 & 19 & 23 & 4 & 20 & 59033.980 & -0.078 & -11.30 & -0.04 \\
\hline 22 & 13 & 9 & 23 & 13 & 10 & 59000.540 & -0.004 & 6.79 & -0.01 \\
\hline 22 & 13 & 10 & 23 & 13 & 11 & 59000.540 & -0.004 & 6.79 & -0.01 \\
\hline
\end{tabular}




\begin{tabular}{|c|c|c|c|c|c|c|c|c|}
\hline 2214 & 8 & 23 & 14 & 9 & 59002.980 & $-\odot .018$ & 9.96 & -0.03 \\
\hline 2214 & 9 & 23 & 14 & 10 & 59002.980 & -0.018 & 9.96 & -0.03 \\
\hline 2215 & 7 & 23 & 15 & 8 & 59005.770 & -0.056 & 13.37 & -0.04 \\
\hline $22 \quad 15$ & 8 & 23 & 15 & 9 & 59005.770 & $-\odot .056$ & 13.37 & $-\odot .04$ \\
\hline 2216 & 6 & 23 & 16 & 7 & 59009.140 & 0.145 & 17.01 & $-\odot .06$ \\
\hline 2216 & 7 & 23 & 16 & 8 & 59009.140 & 0.145 & 17.01 & $-\odot .06$ \\
\hline $22 \quad 17$ & 5 & 23 & 17 & 6 & 59012.430 & $-\odot .049$ & 20.89 & $-\odot .08$ \\
\hline 2217 & 6 & 23 & 17 & 7 & 59012.430 & -0.049 & 20.89 & -0.08 \\
\hline 2218 & 4 & 23 & 18 & 5 & 59016.290 & $\odot .028$ & $25 . \odot \odot$ & -0.10 \\
\hline 2218 & 5 & 23 & 18 & 6 & 59016.290 & 0.028 & $25.0 \odot$ & -0.10 \\
\hline
\end{tabular}

RMS $=0.1731 \quad$ GRAMDET.$=\odot .49 \mathrm{E}-\odot 3$

ROTATIONAL CONSTANTS (MHZ) :
$+-$
11076.834582
1319.765878
1244.882880
$\odot .004577$
QUARTIC DISTORTION CONSTANTS:

$\begin{array}{rrrrrr} & 0.26987550 & -2.56524805 & 29.16152100 & 0.02701800 & 1.20187600 \\ +- & 0.00231036 & 0.00518039 & 0.00000000 & 0.0000000 \odot & 0.0000000 \odot\end{array}$

\section{SIGNIFICANT DIGITS AND CORRELATION MATRIX:}

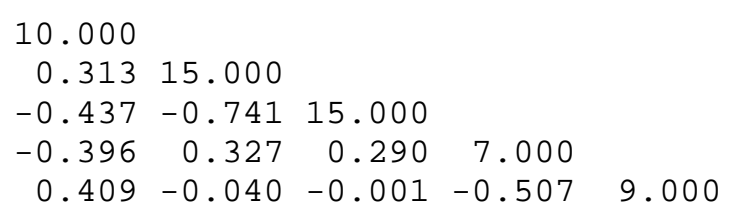

DERIVED CONSTANTS:

KAPPA $=-0.984767$

MOMENTS OF INERTIA AND DEFECT $\left(U^{*} A^{* *} 2\right)$ : $45.62486207382 .93083537405 .96513802-22.59055942$

$\begin{array}{lllll}+- & 0.02060190 & 0.00133590 & 0.00149257 & 0.02180682\end{array}$

STANDARD DISTORTION CONSTANTS (KHZ):
$-107.464594$
$-1.295646$
$-0.863358$
$-\odot .864582$
$-1.079502$
8.966570 
Table 5S: Cyclopropylmethylselenol Conformer V $\left({ }^{82} \mathrm{Se}\right)$. Ground vibrational state.

TOTAL NUMBER OF ACCEPTED TRANSITIONS: 44

\begin{tabular}{|c|c|c|c|c|c|c|c|c|c|}
\hline \multicolumn{6}{|c|}{ TRANSITION } & OBS. FREQ. & $0 .-C$. & \multicolumn{2}{|c|}{ 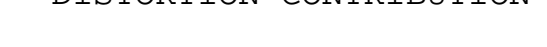 } \\
\hline 19 & 12 & 7 & 20 & 12 & 8 & 50892.340 & -0.102 & $\begin{array}{r}\text { TOTAL } \\
4.79\end{array}$ & $\begin{array}{l}\text { FIXED } \\
-0.02\end{array}$ \\
\hline 19 & 12 & 8 & 20 & 12 & 9 & 50892.340 & -0.102 & 4.79 & -0.02 \\
\hline 19 & 13 & 6 & 20 & 13 & 7 & 50894.230 & 0.019 & 7.14 & -0.03 \\
\hline 19 & 13 & 7 & 20 & 13 & 8 & 50894.230 & $\odot .019$ & 7.14 & $-\odot . \odot 3$ \\
\hline 19 & 14 & 5 & 20 & 14 & 6 & 50896.210 & -0.080 & 9.69 & -0.04 \\
\hline 19 & 14 & 6 & 20 & 14 & 7 & 50896.210 & -0.080 & 9.69 & $-\odot .04$ \\
\hline 19 & 15 & 4 & 20 & 15 & 5 & 50898.550 & $-\odot .098$ & 12.42 & -0.05 \\
\hline 19 & 15 & 5 & 20 & 15 & 6 & 50898.550 & -0.098 & 12.42 & -0.05 \\
\hline 19 & 16 & 3 & 20 & 16 & 4 & 50901.160 & -0.102 & 15.34 & $-\odot .07$ \\
\hline 19 & 16 & 4 & 20 & 16 & 5 & 50901.160 & -0.102 & 15.34 & $-\odot .07$ \\
\hline 20 & 4 & 16 & 21 & 4 & 17 & 53464.130 & 0.459 & -8.67 & -0.03 \\
\hline 20 & 4 & 17 & 21 & 4 & 18 & 53462.100 & -0.058 & -8.66 & $-\odot .02$ \\
\hline 20 & 14 & 6 & 21 & 14 & 7 & 53440.620 & 0.149 & 9.23 & -0.04 \\
\hline 20 & 14 & 7 & 21 & 14 & 8 & 53440.620 & 0.149 & 9.23 & -0.04 \\
\hline 20 & 15 & 5 & 21 & 15 & 6 & 53442.950 & 0.043 & 12.10 & -0.05 \\
\hline 20 & 15 & 6 & 21 & 15 & 7 & 53442.950 & $\odot .043$ & 12.10 & $-\odot .05$ \\
\hline 20 & 16 & 4 & 21 & 16 & 5 & 53445.670 & 0.051 & 15.16 & -0.07 \\
\hline 20 & 16 & 5 & 21 & 16 & 6 & 53445.670 & 0.051 & 15.16 & -0.07 \\
\hline 20 & 17 & 3 & 21 & 17 & 4 & 53448.690 & 0.100 & 18.43 & $-\odot . \odot 8$ \\
\hline 20 & 17 & 4 & 21 & 17 & 5 & 53448.690 & 0.100 & 18.43 & -0.08 \\
\hline 21 & 4 & 17 & 22 & 4 & 18 & 56013.340 & $\odot . \odot 89$ & -10.14 & $-\odot . \odot 4$ \\
\hline 21 & 4 & 18 & 22 & 4 & 19 & 56011.190 & 0.037 & -10.13 & -0.03 \\
\hline 21 & 13 & 8 & 22 & 13 & 9 & 55982.430 & $\odot .051$ & 5.83 & -0.02 \\
\hline 21 & 13 & 9 & 22 & 13 & 10 & 55982.430 & 0.051 & 5.83 & -0.02 \\
\hline 21 & 14 & 7 & 22 & 14 & 8 & 55984.540 & -0.019 & 8.63 & -0.03 \\
\hline 21 & 14 & 8 & 22 & 14 & 9 & 55984.540 & $-\odot .019$ & 8.63 & -0.03 \\
\hline 21 & 15 & 6 & 22 & 15 & 7 & 55986.980 & $-\odot .087$ & 11.63 & -0.05 \\
\hline 21 & 15 & 7 & 22 & 15 & 8 & 55986.980 & -0.087 & 11.63 & -0.05 \\
\hline 21 & 16 & 5 & 22 & 16 & 6 & 55989.750 & -0.122 & 14.85 & -0.06 \\
\hline 21 & 16 & 6 & 22 & 16 & 7 & 55989.750 & -0.122 & 14.85 & -0.06 \\
\hline 21 & 17 & 4 & 22 & 17 & 5 & 55992.860 & -0.095 & 18.27 & -0.08 \\
\hline 21 & 17 & 5 & 22 & 17 & 6 & 55992.860 & -0.095 & 18.27 & -0.08 \\
\hline 22 & 4 & 18 & 23 & 4 & 19 & 58563.300 & -0.108 & -11.76 & -0.06 \\
\hline 22 & 4 & 19 & 23 & 4 & 20 & 58560.530 & -0.014 & -11.75 & $-0.0<$ \\
\hline 22 & 14 & 8 & 23 & 14 & 9 & 58528.590 & 0.040 & 7.88 & $-\odot .03$ \\
\hline 22 & 14 & 9 & 23 & 14 & 10 & 58528.590 & $0.04 \odot$ & 7.88 & -0.03 \\
\hline 22 & 15 & 7 & 23 & 15 & 8 & 58531.120 & -0.003 & 11.03 & $-0.0<$ \\
\hline 22 & 15 & 8 & 23 & 15 & 9 & 58531.120 & -0.003 & 11.03 & -0.0 \\
\hline 22 & 16 & 6 & 23 & 16 & 7 & 58533.920 & -0.097 & 14.39 & -0.0 \\
\hline 22 & 16 & 7 & 23 & 16 & 8 & 58533.920 & $-\odot .097$ & 14.39 & -0.06 \\
\hline 22 & 17 & 5 & 23 & 17 & 6 & 58537.480 & 0.273 & 17.96 & -0.0 \\
\hline 22 & 17 & 6 & 23 & 17 & 7 & 58537.480 & 0.273 & 17.96 & -0.0 \\
\hline 23 & 4 & 19 & 24 & 4 & 20 & 61113.980 & -0.210 & -13.54 & $-\odot . \odot$ \\
\hline 23 & 4 & 20 & 24 & 4 & 21 & 61110.290 & $-\odot . \odot 42$ & -13.52 & $-\odot . \odot$ \\
\hline
\end{tabular}

RMS $=0.1293 \quad$ GRAMDET.$=0.63 \mathrm{E}-\odot 6$ 


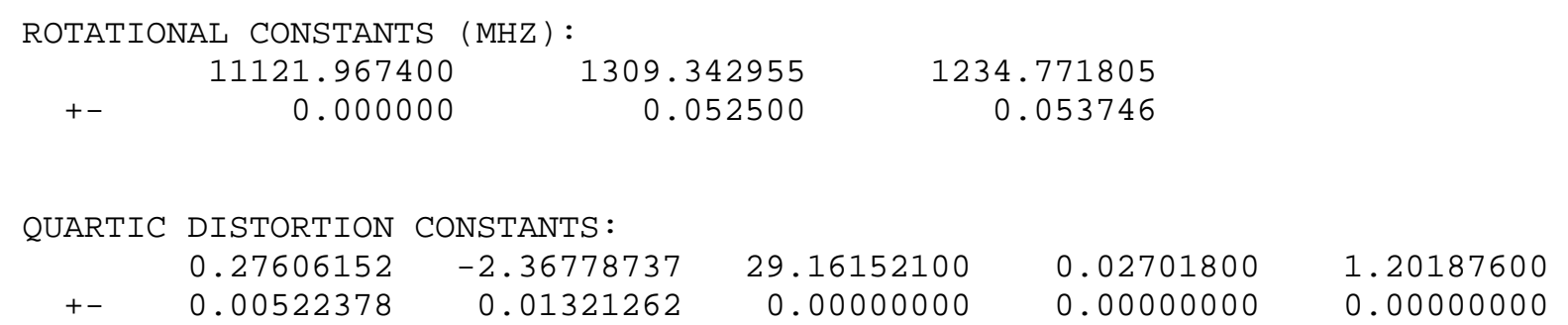

SIGNIFICANT DIGITS AND CORRELATION MATRIX:

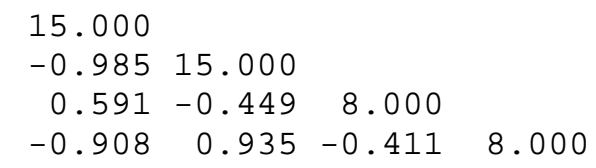

\section{DERIVED CONSTANTS :}

KAPPA $=-\odot .984916$

MOMENTS OF INERTIA AND DEFECT $\left(U^{*} A * * 2\right)$ : $45.43971690385 .97912655 \quad 409.28943137-22.12941208$

+- $\quad 0.00000000 \quad 0.01547627 \quad 0.01781508$

STANDARD DISTORTION CONSTANTS (KHZ):

$\begin{array}{rrr}-108.279181 & -1.320390 & -0.888102 \\ -1.284247 & -1.104246 & 8.546905\end{array}$


Table 6S: Cyclopropylmethylselenol Conformer $V\left(\mathrm{C}_{3} \mathrm{H}_{5} \mathrm{CH}_{2}{ }^{80} \mathrm{SeD}\right)$. Ground vibrational state.

TOTAL NUMBER OF ACCEPTED TRANSITIONS: 77

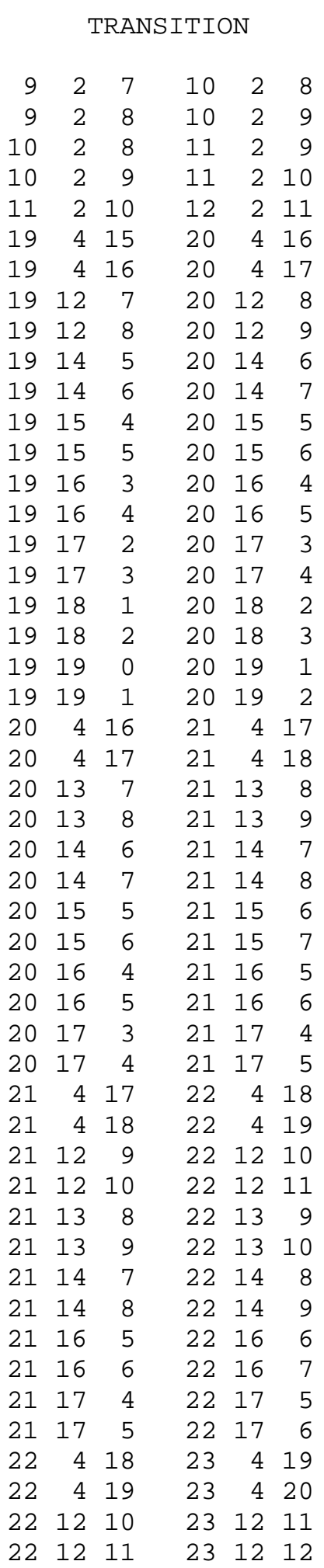

OBS. FREQ . O.-C.

$25621.740-0.041$

$25538.560-0.343$

$28199.330 \quad 0.063$

$28089.040-0.235$

$30638.180-0.464$

$51145.440 \quad 0.088$

$51143.460-0.273$

$51117.710 \quad 0.001$

$51117.710 \quad 0.001$

$51122.140 \quad 0.164$

$51122.140 \quad 0.164$

$51124.680 \quad 0.069$

$51124.680 \quad 0.069$

$51128.080 \quad 0.540$

$51128.080 \quad 0.540$

$51130.480-0.264$

$51130.480-0.264$

$51134.660 \quad 0.449$

$51134.660 \quad 0.449$

$51137.770-0.159$

$51137.770-0.159$

$53706.900 \quad 0.115$

$53704.000-0.504$

$53675.170 \quad 0.100$

$53675.170 \quad 0.100$

$53677.690 \quad 0.251$

$53677.690 \quad 0.251$

$53680.550 \quad 0.393$

$53680.550 \quad 0.393$

$53683.050-0.143$

$53683.050-0.143$

$53686.440-0.085$

$53686.440-0.085$

$56269.220 \quad 0.316$

$56265.280-0.462$

$56228.480 \quad 0.076$

$56228.480 \quad 0.076$

$56230.450 \quad 0.058$

$56230.450 \quad 0.058$

$56232.870 \quad 0.062$

$56232.870 \quad 0.062$

$56238.550-0.190$

$56238.550-0.190$

$56242.160-0.035$

$56242.160-0.035$

$58832.070 \quad 0.304$

$58827.160-0.290$

$58783.570-0.068$

$58783.570-0.068$
DISTORTION CONTRIBUTION

TOTAL

$-1.00$

FIXED

$-0.95$

$-1.39$

$-1.31$

$-1.74$

$-7.76$

$-7.75$

5.93

5.93

11.46

11.46

14.54

14.54

17.84

17.84

21.35

21.35

25.07

25.07

29.00

29.00

$-9.18$

$-9.17$

8. 01

8. 01

11.02

11.02

14.26

14.26

17.72

17.72

21.40

21.40

$-10.75$

$-10.73$

4.35

4.35

7.28

7.28

10.44

10.44

17.45

17.45

21.31

21.31

$-12.48$

$-12.46$

3.33

3.33
$-0.03$

0.02

$-0.05$

0.03

0.04

$-0.02$

$-0.01$

$-0.02$

$-0.02$

$-0.05$

$-0.05$

$-0.06$

$-0.06$

$-0.08$

$-0.08$

$-0.10$

$-0.10$

$-0.11$

$-0.11$

$-0.13$

$-0.13$

$-0.03$

$-0.02$

$-0.03$

$-0.03$

$-0.04$

$-0.04$

$-0.06$

$-0.06$

$-0.08$

$-0.08$

$-0.09$

$-0.09$

$-0.05$

$-0.03$

$-0.01$

$-0.01$

$-0.02$

$-0.02$

$-0.04$

$-0.04$

$-0.07$

$-0.07$

$-0.09$

$-0.09$

$-0.07$

$-0.05$ 


$\begin{array}{rrrrrr}22 & 13 & 9 & 23 & 13 & 10 \\ 22 & 13 & 10 & 23 & 13 & 11 \\ 22 & 14 & 8 & 23 & 14 & 9 \\ 22 & 14 & 9 & 23 & 14 & 10 \\ 22 & 15 & 7 & 23 & 15 & 8 \\ 22 & 15 & 8 & 23 & 15 & 9 \\ 22 & 16 & 6 & 23 & 16 & 7 \\ 22 & 16 & 7 & 23 & 16 & 8 \\ 22 & 17 & 5 & 23 & 17 & 6 \\ 22 & 17 & 6 & 23 & 17 & 7 \\ 22 & 18 & 4 & 23 & 18 & 5 \\ 22 & 18 & 5 & 23 & 18 & 6 \\ 22 & 19 & 3 & 23 & 19 & 4 \\ 22 & 19 & 4 & 23 & 19 & 5 \\ 23 & 12 & 11 & 24 & 12 & 12 \\ 23 & 12 & 12 & 24 & 12 & 13 \\ 23 & 13 & 10 & 24 & 13 & 11 \\ 23 & 13 & 11 & 24 & 13 & 12 \\ 23 & 14 & 9 & 24 & 14 & 10 \\ 23 & 14 & 10 & 24 & 14 & 11 \\ 23 & 15 & 8 & 24 & 15 & 9 \\ 23 & 15 & 9 & 24 & 15 & 10 \\ 23 & 16 & 7 & 24 & 16 & 8 \\ 23 & 16 & 8 & 24 & 16 & 9 \\ 23 & 17 & 6 & 24 & 17 & 7 \\ 23 & 17 & 7 & 24 & 17 & 8 \\ 23 & 18 & 5 & 24 & 18 & 6 \\ 23 & 18 & 6 & 24 & 18 & 7\end{array}$

\begin{tabular}{|c|c|}
\hline & \\
\hline & \\
\hline & \\
\hline & \\
\hline & \\
\hline & \\
\hline & $-\odot .065$ \\
\hline & -0. \\
\hline 720 & -0. \\
\hline & \\
\hline & \\
\hline 3801.370 & \\
\hline & -0.2 \\
\hline 590 & $-\odot$. \\
\hline$\$ 40$ & $-\odot$. \\
\hline 540 & $-\odot$. \\
\hline .970 & 0. \\
\hline .970 & 0.2 \\
\hline .450 & \\
\hline 45 & \\
\hline .240 & 0.0 \\
\hline 46.240 & 0.067 \\
\hline $49.57 \mathrm{C}$ & 0.075 \\
\hline 61349.570 & 0.075 \\
\hline 1353.120 & -0.060 \\
\hline 1353.12 & \\
\hline & \\
\hline & \\
\hline
\end{tabular}

6.39

6.39

9.70

9.70

13.24

13.24

17.03

17.03

21.07

21.07

25.35

25.35

29.87

29.87

2.15

2.15

5.35

5.35

8.79

8.79

12.49

12.49

16.45

16.45

20.66

20.66

25.13

25.13
$-0.02$

$-0.02$

$-0.03$

$-0.03$

$-0.05$

$-0.05$

$-0.07$

$-0.07$

$-0.09$

$-0.09$

$-0.11$

$-0.11$

$-0.13$

$-0.13$

0.01

0.01

$-0.01$

$-0.01$

$-0.02$

$-0.02$

$-0.04$

$-0.04$

$-0.06$

$-0.06$

$-0.08$

$-0.08$

$-0.11$

$-0.11$

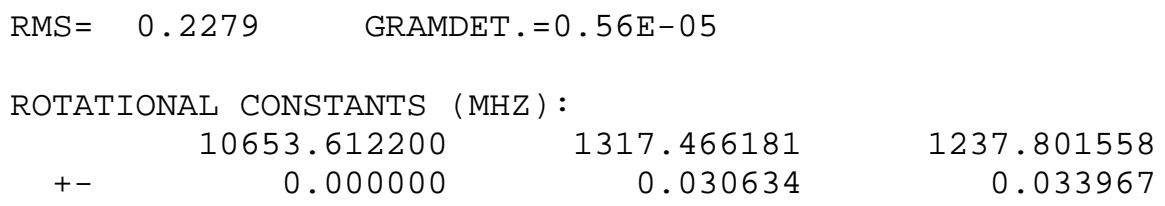

SIGNIFICANT DIGITS AND CORRELATION MATRIX:

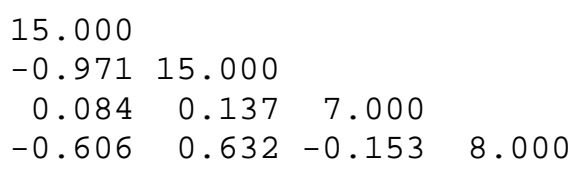

DERIVED CONSTANTS:

$\mathrm{KAPPA}=-0.983079$

MOMENTS OF INERTIA AND DEFECT $(U * A * * 2)$ : $47.43734243 \quad 383.59925854 \quad 408.28761836-22.74898261$
$+$
$\odot .00000 \odot \odot \odot$
○. 00891942
๑. 01120382 
STANDARD DISTORTION CONSTANTS (KHZ):

$\begin{array}{rrr}-107.139056 & -1.396506 & -0.964218 \\ -0.752243 & -1.180362 & 9.078909\end{array}$


Table 7S: Cyclopropylmethylselenol Conformer $V\left({ }^{78} \mathrm{Se}\right)$. Excited vibrational state $78 \mathrm{Va}$.

TOTAL NUMBER OF ACCEPTED TRANSITIONS: 32

\begin{tabular}{|c|c|c|c|c|c|c|c|c|c|}
\hline & & TRAI & ITIC & & & OBS. FREQ. & $0 .-C$. & DIST & UTION \\
\hline & & & & & & & & TOTAL & FIXED \\
\hline 19 & 11 & 8 & 20 & 11 & 9 & 51650.840 & -0.138 & 3.28 & -0.01 \\
\hline 19 & 11 & 9 & 20 & 11 & 10 & 51650.840 & -0.138 & 3.28 & -0.01 \\
\hline 19 & 13 & 6 & 20 & 13 & 7 & 51654.250 & -0.198 & 8.14 & -0.03 \\
\hline 19 & 13 & 7 & 20 & 13 & 8 & 51654.250 & -0.198 & 8.14 & -0.03 \\
\hline 19 & 15 & 4 & 20 & 15 & 5 & 51659.120 & -0.129 & 13.81 & -0.06 \\
\hline 19 & 15 & 5 & 20 & 15 & 6 & 51659.120 & -0.129 & 13.81 & -0.06 \\
\hline 20 & 4 & 16 & 21 & 4 & 17 & 54262.510 & 0.264 & -8.77 & -0.03 \\
\hline 20 & 4 & 17 & 21 & 4 & 18 & 54260.440 & -0.170 & -8.77 & -0.02 \\
\hline 20 & 12 & 8 & 21 & 12 & 9 & 54234.770 & 0.146 & 4.92 & -0.01 \\
\hline 20 & 12 & 9 & 21 & 12 & 10 & 54234.770 & 0.146 & 4.92 & $-\odot .01$ \\
\hline 20 & 14 & 6 & 21 & 14 & 7 & 54239.150 & $\odot .257$ & 10.45 & -0.04 \\
\hline 20 & 14 & 7 & 21 & 14 & 8 & 54239.150 & 0.257 & 10.45 & -0.04 \\
\hline 20 & 15 & 5 & 21 & 15 & 6 & 54241.660 & 0.133 & 13.54 & -0.05 \\
\hline 20 & 15 & 6 & 21 & 15 & 7 & 54241.660 & 0.133 & 13.54 & -0.05 \\
\hline 21 & 4 & 17 & 22 & 4 & 18 & 56849.930 & -0.080 & -10.27 & $-\odot .04$ \\
\hline 21 & 4 & 18 & 22 & 4 & 19 & 56847.580 & -0.163 & -10.26 & $-\odot .03$ \\
\hline 21 & 13 & 8 & 22 & 13 & 9 & 56818.800 & 0.177 & 6.88 & $-\odot .02$ \\
\hline 21 & 13 & 9 & 22 & 13 & 10 & 56818.800 & 0.177 & 6.88 & $-\odot .02$ \\
\hline 21 & 15 & 6 & 22 & 15 & 7 & 56823.740 & $\odot .035$ & 13.12 & -0.05 \\
\hline 21 & 15 & 7 & 22 & 15 & 8 & 56823.740 & 0.035 & 13.12 & -0.05 \\
\hline 21 & 16 & 5 & 22 & 16 & 6 & 56826.770 & $\odot .034$ & 16.58 & $-\odot . \odot 7$ \\
\hline 21 & 16 & 6 & 22 & 16 & 7 & 56826.770 & $\odot .034$ & 16.58 & -0.07 \\
\hline 22 & 4 & 18 & 23 & 4 & 19 & 59438.460 & 0.081 & -11.93 & $-\odot .06$ \\
\hline 22 & 4 & 19 & 23 & 4 & 20 & 59435.330 & 0.047 & -11.91 & -0.04 \\
\hline 22 & 13 & 9 & 23 & 13 & 10 & 59400.290 & -0.287 & 6.03 & $-\odot .01$ \\
\hline 22 & 13 & 10 & 23 & 13 & 11 & 59400.290 & -0.287 & 6.03 & $-\odot .01$ \\
\hline 22 & 14 & 8 & 23 & 14 & 9 & 59403.100 & 0.110 & 9.18 & -0.03 \\
\hline 22 & 14 & 9 & 23 & 14 & 10 & 59403.100 & 0.110 & 9.18 & $-\odot .03$ \\
\hline 22 & 16 & 6 & 23 & 16 & 7 & 59408.980 & 0.075 & 16.17 & -0.06 \\
\hline 22 & 16 & 7 & 23 & 16 & 8 & 59408.980 & $\odot .075$ & 16.17 & -0.06 \\
\hline 22 & 17 & 5 & 23 & 17 & 6 & 59412.140 & -0.209 & 20.01 & $-\odot . \odot \varepsilon$ \\
\hline 22 & 17 & 6 & 23 & 17 & 7 & 59412.140 & -0.209 & 20.01 & $-\odot . \odot \varepsilon$ \\
\hline
\end{tabular}

RMS $=0.1747 \quad$ GRAMDET.$=0.67 \mathrm{E}-06$

ROTATIONAL CONSTANTS (MHZ):

$\begin{array}{rrrr}11052.700000 & 1328.799350 & 1253.268961 \\ +- & 0.000000 & 0.076720 & 0.077001\end{array}$

QUARTIC DISTORTION CONSTANTS:

$\begin{array}{rrrrrr} & 0.28205435 & -2.54392216 & 29.16152100 & 0.02701800 & 1.20187600 \\ +- & 0.00892494 & 0.02068700 & 0.00000000 & 0.0000000 \odot & 0.0000000 \odot\end{array}$




\section{SIGNIFICANT DIGITS AND CORRELATION MATRIX:}

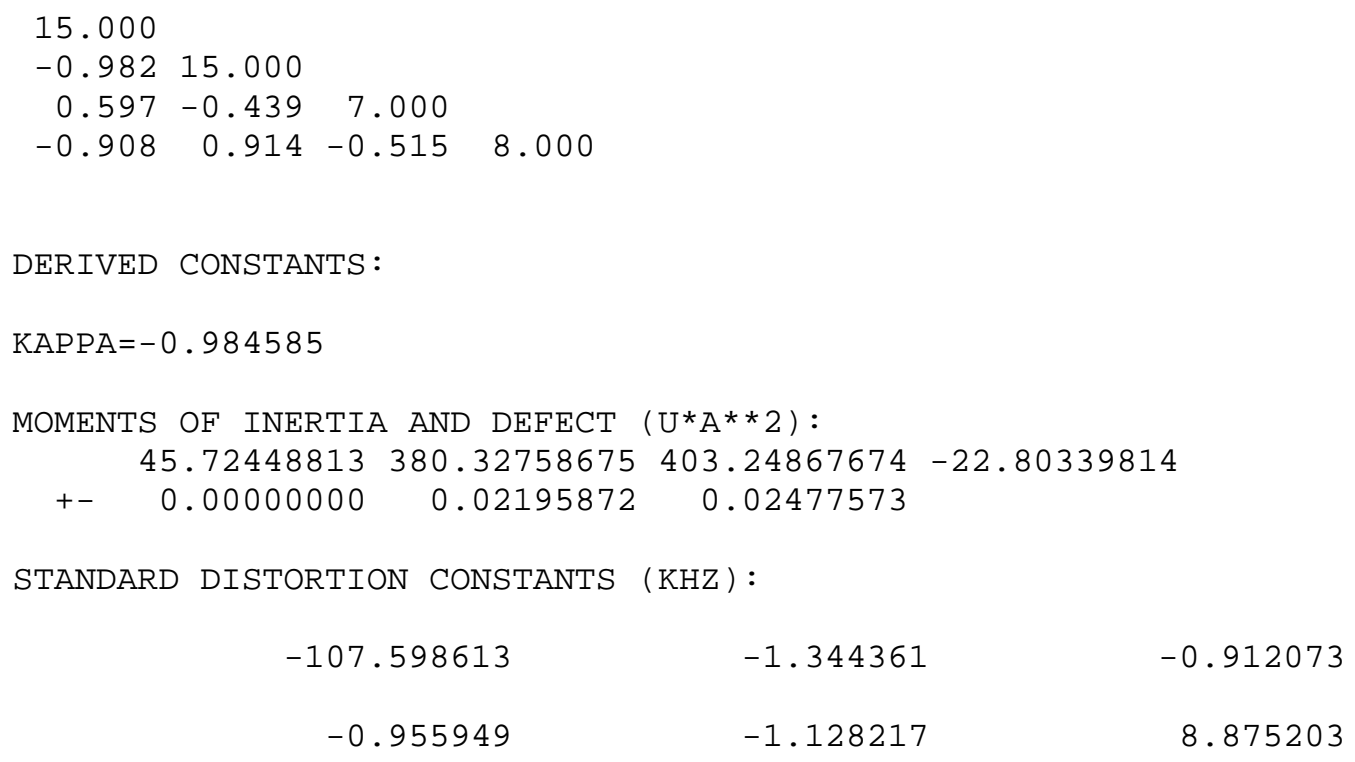

8.875203 
Table 8S: Cyclopropylmethylselenol Conformer $\mathrm{V}\left({ }^{78} \mathrm{Se}\right)$. Excited vibrational state $78 \mathrm{Vb}$.

TOTAL NUMBER OF ACCEPTED TRANSITIONS: 40

$\begin{array}{rrrrrr} & & \text { TRANSITION } & \\ & & & & & \\ 20 & 13 & 7 & 21 & 13 & 8 \\ 20 & 13 & 8 & 21 & 13 & 9 \\ 20 & 14 & 6 & 21 & 14 & 7 \\ 20 & 14 & 7 & 21 & 14 & 8 \\ 20 & 15 & 5 & 21 & 15 & 6 \\ 20 & 15 & 6 & 21 & 15 & 7 \\ 20 & 16 & 4 & 21 & 16 & 5 \\ 20 & 16 & 5 & 21 & 16 & 6 \\ 20 & 17 & 3 & 21 & 17 & 4 \\ 20 & 17 & 4 & 21 & 17 & 5 \\ 20 & 18 & 2 & 21 & 18 & 3 \\ 20 & 18 & 3 & 21 & 18 & 4 \\ 20 & 19 & 1 & 21 & 19 & 2 \\ 20 & 19 & 2 & 21 & 19 & 3 \\ 21 & 12 & 9 & 22 & 12 & 10 \\ 21 & 12 & 10 & 22 & 12 & 11 \\ 21 & 13 & 8 & 22 & 13 & 9 \\ 21 & 13 & 9 & 22 & 13 & 10 \\ 21 & 14 & 7 & 22 & 14 & 8 \\ 21 & 14 & 8 & 22 & 14 & 9 \\ 21 & 15 & 6 & 22 & 15 & 7 \\ 21 & 15 & 7 & 22 & 15 & 8 \\ 21 & 16 & 5 & 22 & 16 & 6 \\ 21 & 16 & 6 & 22 & 16 & 7 \\ 22 & 13 & 9 & 23 & 13 & 10 \\ 22 & 13 & 10 & 23 & 13 & 11 \\ 22 & 14 & 8 & 23 & 14 & 9 \\ 22 & 14 & 9 & 23 & 14 & 10 \\ 22 & 15 & 7 & 23 & 15 & 8 \\ 22 & 15 & 8 & 23 & 15 & 9 \\ 22 & 16 & 6 & 23 & 16 & 7 \\ 22 & 16 & 7 & 23 & 16 & 8 \\ 22 & 17 & 5 & 23 & 17 & 6 \\ 22 & 17 & 6 & 23 & 17 & 7 \\ 22 & 18 & 4 & 23 & 18 & 5 \\ 22 & 18 & 5 & 23 & 18 & 6 \\ 22 & 19 & 3 & 23 & 19 & 4 \\ 22 & 19 & 4 & 23 & 19 & 5 \\ 22 & 20 & 2 & 23 & 20 & 3 \\ 22 & 20 & 3 & 23 & 20 & 4 \\ & & & & & \end{array}$

\begin{tabular}{|c|c|}
\hline REQ . & $0 .-\mathrm{C}$ \\
\hline 4140.020 & \\
\hline 4140.020 & \\
\hline 4142.29 & \\
\hline 42.290 & \\
\hline 4.930 & \\
\hline 44.930 & \\
\hline 48.020 & \\
\hline 48.020 & \\
\hline 51.390 & \\
\hline 51.390 & 0.036 \\
\hline 55.120 & \\
\hline 4155.120 & 0.050 \\
\hline 4159.020 & \\
\hline 4159.020 & -0.058 \\
\hline 6715.470 & \\
\hline 6715.470 & -0.040 \\
\hline 6717.190 & -0.106 \\
\hline 6717.190 & -0.106 \\
\hline 6719.500 & -0.097 \\
\hline 6719.500 & -0.097 \\
\hline 6722.180 & -0.165 \\
\hline 6722.180 & -0.165 \\
\hline 5725.400 & -0.089 \\
\hline 6725.400 & -0.089 \\
\hline 9294.670 & 0.110 \\
\hline 294.670 & 0.110 \\
\hline 9296.930 & 0.063 \\
\hline 9296.930 & 0.063 \\
\hline 9299.640 & -0.019 \\
\hline 9299.640 & -0.019 \\
\hline 9302.850 & -0.031 \\
\hline 9302.850 & -0.031 \\
\hline 9306.590 & 0.097 \\
\hline 9306.590 & $\odot .097$ \\
\hline 9310.520 & $\odot .053$ \\
\hline 9310.520 & 0.053 \\
\hline 9314.730 & -0.052 \\
\hline 9314.730 & -0.052 \\
\hline 9319.430 & 0.010 \\
\hline 9319.430 & \\
\hline
\end{tabular}

DISTORTION CONTRIBUTION

TOTAL

6.33

6.33

9.50

9.50

12.92

12.92

16.56

16.56

20.45

20.45

24.56

24.56

28.92

28.92

2.16

2.16

5.24

5.24

8.57

8.57

12.15

12.15

15.97

15.97

3.96

3.96

7.44

7.44

11.18

11.18

15.18

15.18

19.43

19.43

23.94

23.94

28.71

28.71

33.73

33.73
FIXED

$-0.03$

$-0.03$

$-0.05$

$-0.05$

$-0.07$

$-0.07$

$-0.09$

$-0.09$

$-0.11$

$-0.11$

$-0.13$

$-0.13$

$-0.15$

$-0.15$

$-0.01$

$-0.01$

$-0.02$

$-0.02$

$-0.04$

$-0.04$

$-0.06$

$-0.06$

$-0.08$

$-0.08$

$-0.02$

$-0.02$

$-0.04$

$-0.04$

$-0.06$

$-0.06$

$-0.08$

$-0.08$

$-0.10$

$-0.10$

$-0.12$

$-0.12$

$-0.15$

$-0.15$

$-0.18$

$-0.18$

RMS $=0.0795 \quad$ GRAMDET.$=\odot .28 \mathrm{E}-09$

ROTATIONAL CONSTANTS (MHZ):

$\begin{array}{rrrr}11052.700000 & 1336.486725 & 1240.879710 \\ +- & 0.000000 & 0.712980 & 0.723414\end{array}$




$\begin{array}{rrrrrr}\text { QUARTIC } & \text { DISTORTION } & \text { CONSTANTS: } & & & \\ & 0.36804214 & -2.81656298 & 29.16152100 & 0.02701800 & 1.20187600 \\ +- & 0.00548937 & 0.01500913 & 0.00000000 & 0.00000000 & 0.00000000\end{array}$

SIGNIFICANT DIGITS AND CORRELATION MATRIX:

16.000

$-1.00016 .000$

$0.724-0.717 \quad 8.000$

$-0.964 \quad 0.964-0.721 \quad 9.00 \odot$

DERIVED CONSTANTS:

KAPPA $=-\odot .980512$

MOMENTS OF INERTIA AND DEFECT $\left(U^{*} A * * 2\right)$ : $45.72448813 \quad 378.13997004 \quad 407.27481154-16.58964663$

+- $\quad 0.00000000 \quad 0.20172771 \quad 0.23743488$

STANDARD DISTORTION CONSTANTS (KHZ):

$\begin{array}{rrr}-106.852001 & -1.688313 & -1.256025 \\ -0.754619 & -1.472169 & 9.076533\end{array}$


Table 9S: Cyclopropylmethylselenol Conformer V $\left({ }^{80} \mathrm{Se}\right)$. Excited vibrational state $80 \mathrm{Va}$.

TOTAL NUMBER OF ACCEPTED TRANSITIONS: 54

\begin{tabular}{|c|c|c|c|c|c|c|c|c|c|}
\hline \multicolumn{6}{|c|}{ TRANSITION } & \multirow{3}{*}{$\begin{array}{l}\text { OBS . FREQ. } \\
46104.350\end{array}$} & \multirow{3}{*}{$\begin{array}{l}0 .-C . \\
0.216\end{array}$} & \multirow{2}{*}{\multicolumn{2}{|c|}{$\begin{array}{ll}\text { TOTAL } & \text { FIXED }\end{array}$}} \\
\hline & & & & & & & & & \\
\hline 17 & 13 & 4 & 18 & 13 & 5 & & & 9.60 & -0.03 \\
\hline 17 & 13 & 5 & 18 & 13 & 6 & 46104.350 & 0.216 & 9.60 & -0.03 \\
\hline 17 & 14 & 3 & 18 & 14 & 4 & 46106.200 & $-\odot .067$ & $12 . \odot 8$ & $-\odot .05$ \\
\hline 17 & 14 & 4 & 18 & 14 & 5 & 46106.200 & $-\odot .067$ & $12 . \odot 8$ & -0.05 \\
\hline 17 & 15 & 2 & 18 & 15 & 3 & 46108.510 & -0.140 & 14.73 & $-\odot .06$ \\
\hline 17 & 15 & 3 & 18 & 15 & 4 & 46108.510 & -0.140 & 14.73 & $-\odot .06$ \\
\hline 17 & 16 & 1 & 18 & 16 & 2 & 46111.200 & $-\odot .066$ & 17.57 & $-\odot .07$ \\
\hline 17 & 16 & 2 & 18 & 16 & 3 & 46111.200 & $-\odot .066$ & 17.57 & $-\odot .07$ \\
\hline 18 & 13 & 5 & 19 & 13 & 6 & 48664.740 & -0.323 & 9.43 & -0.03 \\
\hline 18 & 13 & 6 & 19 & 13 & 7 & 48664.740 & -0.323 & 9.43 & $-\odot . \odot 3$ \\
\hline 18 & 14 & 4 & 19 & 14 & 5 & 48666.720 & -0.554 & 12.04 & $-\odot .04$ \\
\hline 18 & 14 & 5 & 19 & 14 & 6 & 48666.720 & -0.554 & 12.04 & $-\odot .04$ \\
\hline 19 & 15 & 4 & 20 & 15 & 5 & 51230.980 & 0.198 & 14.84 & -0.05 \\
\hline 19 & 15 & 5 & 20 & 15 & 6 & 51230.980 & $\odot .198$ & 14.84 & -0.05 \\
\hline 19 & 16 & 3 & 20 & 16 & 4 & 51233.650 & $\odot .020$ & 18.00 & $-\odot .07$ \\
\hline 19 & 16 & 4 & 20 & 16 & 5 & 51233.650 & $\odot .020$ & $18.0 \odot$ & $-\odot .07$ \\
\hline 19 & 17 & 2 & 20 & 17 & 3 & 51236.840 & 0.105 & 21.36 & -0.09 \\
\hline 19 & 17 & 3 & 20 & 17 & 4 & 51236.840 & 0.105 & 21.36 & $-\odot .09$ \\
\hline 19 & 18 & 1 & 20 & 18 & 2 & 51240.390 & 0.306 & 24.92 & -0.10 \\
\hline 19 & 18 & 2 & 20 & 18 & 3 & $51240.39 \odot$ & $\odot .306$ & 24.92 & -0.10 \\
\hline 20 & 4 & 16 & 21 & 4 & 17 & 53811.220 & $\odot .258$ & -7.71 & -0.03 \\
\hline 20 & 4 & 17 & 21 & 4 & 18 & 53809.530 & ๑. 098 & -7.70 & $-\odot .02$ \\
\hline 20 & 13 & 7 & 21 & 13 & 8 & 53786.930 & $\odot .215$ & 8.73 & $-\odot .02$ \\
\hline 20 & 13 & 8 & 21 & 13 & 9 & 53786.930 & 0.215 & 8.73 & -0.02 \\
\hline 20 & 14 & 6 & 21 & 14 & 7 & 53789.200 & 0.139 & 11.61 & -0.04 \\
\hline 20 & 14 & 7 & 21 & 14 & 8 & 53789.200 & $\odot .139$ & 11.61 & $-\odot .04$ \\
\hline 20 & 15 & 5 & 21 & 15 & 6 & 53791.790 & $\odot .064$ & 14.71 & $-\odot .05$ \\
\hline 20 & 15 & 6 & 21 & 15 & 7 & 53791.790 & 0.064 & 14.71 & -0.05 \\
\hline 20 & 16 & 4 & 21 & 16 & 5 & 53794.790 & 0.107 & 18.03 & $-\odot .07$ \\
\hline 20 & 16 & 5 & 21 & 16 & 6 & $53794.79 \odot$ & 0.107 & 18.03 & $-\odot .07$ \\
\hline 20 & 17 & 3 & 21 & 17 & 4 & $53798 . \odot 4 \odot$ & $\odot .125$ & 21.56 & $-\odot . \odot 8$ \\
\hline 20 & 17 & 4 & 21 & 17 & 5 & 53798.040 & 0.125 & 21.56 & $-\odot .08$ \\
\hline 20 & 18 & 2 & 21 & 18 & 3 & 53801.400 & $-\odot . \odot \odot 9$ & 25.30 & -0.10 \\
\hline 20 & 18 & 3 & 21 & 18 & 4 & 53801.400 & $-\odot . \odot \odot 9$ & 25.30 & -0.10 \\
\hline 21 & 4 & 17 & 22 & 4 & 18 & 56377.150 & -0.031 & -9.05 & $-\odot .04$ \\
\hline 21 & 4 & 18 & 22 & 4 & 19 & 56375.030 & -0.029 & -9.04 & -0.03 \\
\hline 21 & 13 & 8 & 22 & 13 & 9 & 56347.520 & ๑. 088 & 8.19 & $-\odot .02$ \\
\hline 21 & 13 & 9 & 22 & 13 & 10 & 56347.520 & ๑. 088 & 8.19 & $-\odot .02$ \\
\hline 21 & 14 & 7 & 22 & 14 & 8 & 56349.890 & 0.055 & 11.21 & -0.03 \\
\hline 21 & 14 & 8 & 22 & 14 & 9 & 56349.890 & 0.055 & 11.21 & -0.03 \\
\hline 21 & 15 & 6 & 22 & 15 & 7 & 56352.590 & $\odot .008$ & 14.46 & -0.05 \\
\hline 21 & 15 & 7 & 22 & 15 & 8 & 56352.590 & 0.008 & 14.46 & -0.05 \\
\hline 21 & 17 & 4 & 22 & 17 & 5 & 56358.750 & -0.250 & 21.63 & $-\odot . \odot 8$ \\
\hline 21 & 17 & 5 & 22 & 17 & 6 & 56358.750 & $-\odot .250$ & 21.63 & $-\odot . \odot 8$ \\
\hline 22 & 4 & 18 & 23 & 4 & 19 & 58943.940 & -0.052 & -10.53 & -0.06 \\
\hline 22 & 4 & 19 & 23 & 4 & 20 & 58940.910 & $-\odot .185$ & -10.52 & $-\odot .04$ \\
\hline 22 & 13 & 9 & 23 & 13 & 10 & 58908.140 & $\odot .069$ & 7.52 & -0.01 \\
\hline 22 & 13 & 10 & 23 & 13 & 11 & 58908.140 & 0.069 & 7.52 & -0.01 \\
\hline 22 & 14 & 8 & 23 & 14 & 9 & 58910.500 & $-\odot .023$ & 10.68 & -0.03 \\
\hline
\end{tabular}




\begin{tabular}{|c|c|c|c|c|c|c|c|}
\hline 2214 & 9 & $\begin{array}{lll}3 & 14 & 10\end{array}$ & 58910.500 & -0.023 & \multicolumn{2}{|c|}{10.68} & -0.03 \\
\hline 2215 & 23 & $\begin{array}{lll}3 & 15 & 8\end{array}$ & 58913.300 & -0.046 & \multicolumn{2}{|c|}{14.08} & -0.04 \\
\hline 2215 & 8 & 315 & 58913.300 & $-\odot .046$ & \multicolumn{2}{|c|}{14.08} & $-\odot .04$ \\
\hline 2217 & 23 & 17 & 58919.700 & -0.284 & \multicolumn{2}{|c|}{21.57} & $-\odot . \odot 8$ \\
\hline 2217 & 6 & 17 & 58919.700 & -0.284 & \multicolumn{2}{|c|}{21.57} & -0.08 \\
\hline RMS $=$ & \multicolumn{2}{|c|}{0.1945} & $\mathrm{~T} .=0.11 \mathrm{E}-05$ & & & & \\
\hline \multicolumn{8}{|c|}{ ROTATIONAL CONSTANTS (MHZ): } \\
\hline \multirow[b]{2}{*}{+-} & \multicolumn{2}{|c|}{11076.800000} & \multicolumn{2}{|c|}{1317.540503} & \multicolumn{2}{|c|}{1243.059405} & \\
\hline & & .000000 & 0.06 & 2204 & \multicolumn{2}{|c|}{0.066871} & \\
\hline \multirow[t]{2}{*}{ QUARTIC } & \multicolumn{3}{|c|}{ DISTORTION CONSTANTS: } & & & & \\
\hline & 0.2536 & $5520 \quad-$ & .55696511 & 29.1615 & 100 & $\odot .02701800$ & 1.20187600 \\
\hline+- & $\odot .0050$ & 9831 & 01442698 & $\odot . \odot \odot \odot \odot$ & & $\odot .0 \odot \odot \odot \odot \odot \odot \odot$ & $\odot . \odot \odot \odot \odot \odot \odot \odot \odot$ \\
\hline
\end{tabular}

\section{SIGNIFICANT DIGITS AND CORRELATION MATRIX:}

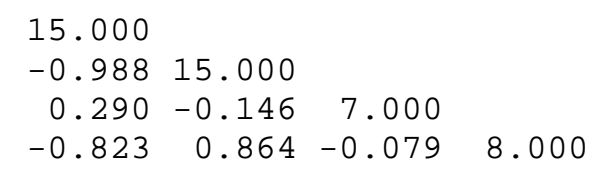

DERIVED CONSTANTS:

KAPPA $=-\odot .984852$

MOMENTS OF INERTIA AND DEFECT $(U * A * * 2)$ : $\begin{array}{lllll}45.62500451 & 383.57761960 & 406.56065843 & -22.64196568\end{array}$

$\begin{array}{llll}+- & 0.00000000 & 0.01810942 & 0.02187126\end{array}$

STANDARD DISTORTION CONSTANTS (KHZ):
$-107.432844$
$-1.230765$
$-\odot .798477$
$-0.816267$
$-1.014621$
9. 014885 
Table 10S: Cyclopropylmethylselenol Conformer V $\left({ }^{80} \mathrm{Se}\right)$. Excited vibrational state $80 \mathrm{Vb}$.

TOTAL NUMBER OF ACCEPTED TRANSITIONS: 56

\begin{tabular}{|c|c|c|c|c|c|}
\hline & & & TIC & & \\
\hline 19 & 13 & 6 & 20 & 13 & 7 \\
\hline 19 & 13 & 7 & 20 & 13 & 8 \\
\hline 19 & 14 & 5 & 20 & 14 & 6 \\
\hline 19 & 14 & 6 & 20 & 14 & 7 \\
\hline 19 & 15 & 4 & 20 & 15 & 5 \\
\hline 19 & 15 & 5 & 20 & 15 & 6 \\
\hline 19 & 16 & 3 & 20 & 16 & 4 \\
\hline 19 & 16 & 4 & 20 & 16 & 5 \\
\hline 19 & 17 & 2 & 20 & 17 & 3 \\
\hline 19 & 17 & 3 & 20 & 17 & 4 \\
\hline 19 & 18 & 1 & 20 & 18 & 2 \\
\hline 19 & 18 & 2 & 20 & 18 & 3 \\
\hline 20 & 13 & 7 & 21 & 13 & 8 \\
\hline 20 & 13 & 8 & 21 & 13 & 9 \\
\hline 20 & 15 & 5 & 21 & 15 & 6 \\
\hline 20 & 15 & 6 & 21 & 15 & 7 \\
\hline 20 & 16 & 4 & 21 & 16 & 5 \\
\hline 20 & 16 & 5 & 21 & 16 & 6 \\
\hline 20 & 17 & 3 & 21 & 17 & 4 \\
\hline 20 & 17 & 4 & 21 & 17 & 5 \\
\hline 20 & 18 & 2 & 21 & 18 & 3 \\
\hline 20 & 18 & 3 & 21 & 18 & 4 \\
\hline 20 & 19 & 1 & 21 & 19 & 2 \\
\hline 20 & 19 & 2 & 21 & 19 & 3 \\
\hline 21 & 13 & 8 & 22 & 13 & 9 \\
\hline 21 & 13 & 9 & 22 & 13 & 10 \\
\hline 21 & 14 & 7 & 22 & 14 & 8 \\
\hline 21 & 14 & 8 & 22 & 14 & 9 \\
\hline 21 & 15 & 6 & 22 & 15 & 7 \\
\hline 21 & 15 & 7 & 22 & 15 & 8 \\
\hline 21 & 16 & 5 & 22 & 16 & 6 \\
\hline 21 & 16 & 6 & 22 & 16 & 7 \\
\hline 21 & 17 & 4 & 22 & 17 & 5 \\
\hline 21 & 17 & 5 & 22 & 17 & 6 \\
\hline 21 & 18 & 3 & 22 & 18 & 4 \\
\hline 21 & 18 & 4 & 22 & 18 & 5 \\
\hline 21 & 19 & 2 & 22 & 19 & 3 \\
\hline 21 & 19 & 3 & 22 & 19 & 4 \\
\hline 21 & 20 & 1 & 22 & 20 & 2 \\
\hline 21 & 20 & 2 & 22 & 20 & 3 \\
\hline 21 & 21 & $\odot$ & 22 & 21 & 1 \\
\hline 21 & 21 & 1 & 22 & 21 & 2 \\
\hline 22 & 12 & 10 & 23 & 12 & 11 \\
\hline 22 & 12 & 11 & 23 & 12 & 12 \\
\hline 22 & 13 & 9 & 23 & 13 & 10 \\
\hline 22 & 13 & 10 & 23 & 13 & 11 \\
\hline 22 & 14 & 8 & 23 & 14 & 9 \\
\hline 22 & 14 & 9 & 23 & 14 & 10 \\
\hline 22 & 15 & 7 & 23 & 15 & \\
\hline
\end{tabular}

OBS. FREQ. O. - C.

$51147.210 \quad 0.159$

$51147.210 \quad 0.159$

$51149.230 \quad 0.101$

$51149.230 \quad 0.101$

$51151.700 \quad 0.136$

$51151.700 \quad 0.136$

$51154.230-0.089$

$51154.230-0.089$

$51157.090-0.280$

$51157.090-0.280$

$51160.440-0.258$

$51160.440-0.258$

$53704.200 \quad 0.166$

$53704.200 \quad 0.166$

$53708.600-0.025$

$53708.600-0.025$

$53711.170-0.295$

$53711.170-0.295$

$53714.640 \quad 0.016$

$53714.640 \quad 0.016$

$53718.110 \quad 0.028$

$53718.110 \quad 0.028$

$53721.850 \quad 0.029$

$53721.850 \quad 0.029$

$56261.150 \quad 0.187$

$56261.150 \quad 0.187$

$56263.140 \quad 0.066$

$56263.140 \quad 0.066$

56265.740

0.128

56265.740

0.128

56268.640

0.112

56268.640

0.112

56271.800

0.011

56271.800

0.011

56275.370

$\odot .0 \odot \odot$

56275.370

0.000

56279.300

$\odot .047$

56279.300

0.047

56283.450

0.026

$56283.450 \quad 0.026$

$56288.050 \quad 0.178$

$56288.050 \quad 0.178$

$58816.100-0.168$

$58816.100-0.168$

$58817.700-0.135$

$58817.700-0.135$

$58819.870-0.074$

$58819.870-0.074$

$58822.500-0.019$
DISTORTION CONTRIBUTION

TOTAL

8.84

8.84

11.68

11.68

14.72

14.72

17.97

17.97

21.44

21.44

25.11

25.11

8.33

8.33

14.50

14.50

17.92

17.92

21.55

21.55

25.41

25.41

29.49

29.49

7.67

7.67

10.79

10.79

14.14

14.14

17.72

17.72

21.53

21.53

25.57

25.57

29.84

29.84

34.34

34.34

39.08

39.08

3.85

3.85

6.87

6.87

10.13

10.13

13.63
FIXED

$-0.04$

$-0.04$

$-0.05$

$-0.05$

$-0.07$

$-0.07$

$-0.09$

$-0.09$

$-0.11$

$-0.11$

$-0.13$

$-0.13$

$-0.03$

$-0.03$

$-0.07$

$-0.07$

$-0.09$

$-0.09$

$-0.11$

$-0.11$

$-0.13$

$-0.13$

$-0.15$

$-0.15$

$-0.02$

$-0.02$

$-0.04$

$-0.04$

$-0.06$

$-0.06$

$-0.08$

$-0.08$

$-0.10$

$-0.10$

$-0.13$

$-0.13$

$-0.15$

$-0.15$

$-0.18$

$-0.18$

$-0.21$

$-0.21$

$-0.02$

$-0.02$

$-0.03$

$-0.03$

$-0.05$ 


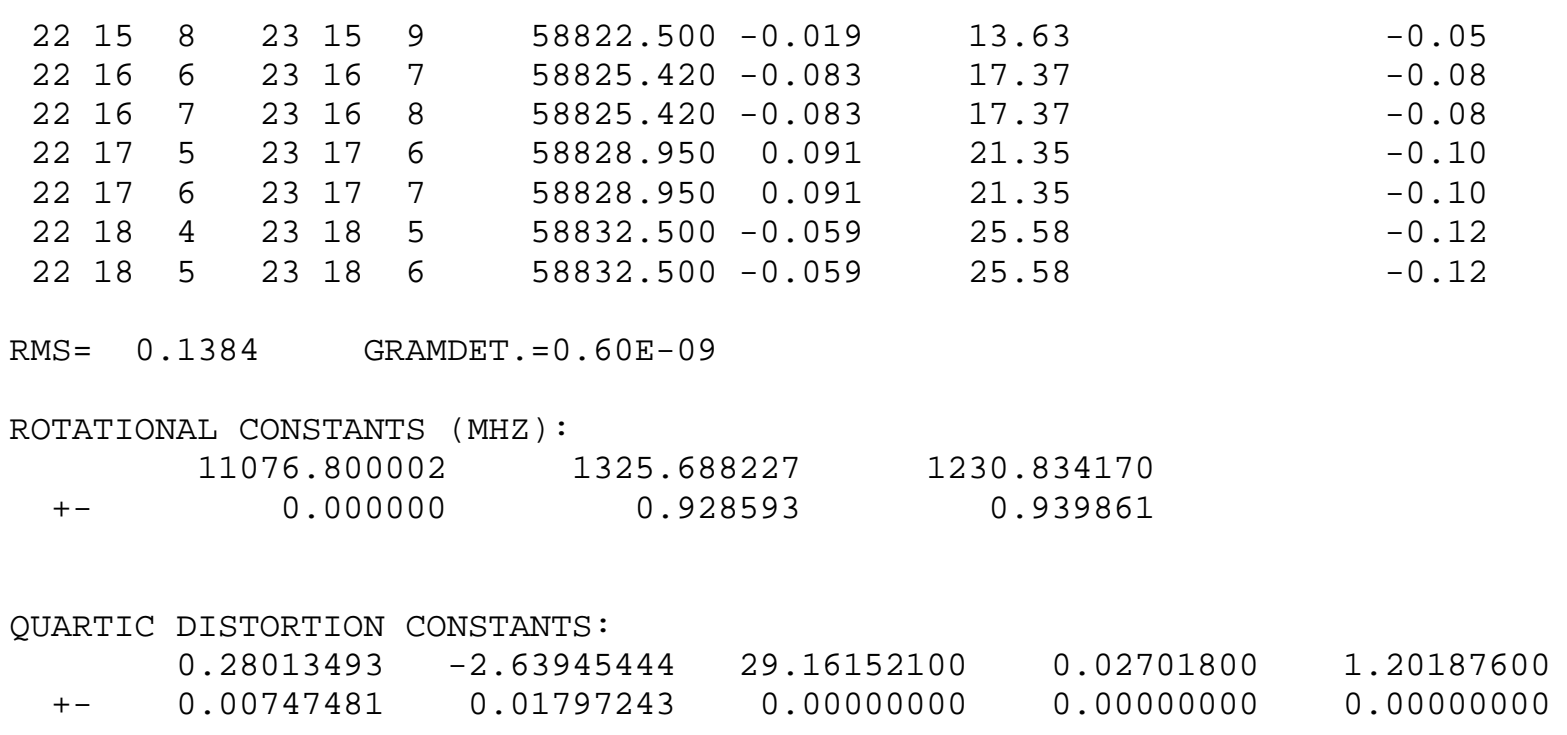

\section{SIGNIFICANT DIGITS AND CORRELATION MATRIX:}

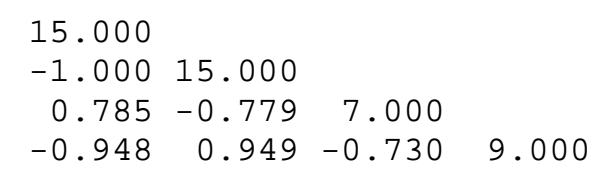

\section{DERIVED CONSTANTS :}

KAPPA $=-\odot .980732$

MOMENTS OF INERTIA AND DEFECT $\left(U^{*} A * * 2\right)$ : $45.62500451381 .22013901410 .59881381-16.24632971$
$+$
0.00000000
0.26702973
0.31353190

STANDARD DISTORTION CONSTANTS (KHZ):
$-107.208806$
$-1.336684$
$-0.904396$
$-\odot .757207$
$-1.120540$
9.073945 
TABLE 11S: Geometries of Four Conformers of Cyclopropylmethylselenol from ab initio Geometry Optimizations at the MP2/aug-cc-pVTZ Level of Theory

\begin{tabular}{|c|c|c|c|c|}
\hline $\begin{array}{l}\text { Structural } \\
\text { parameter }\end{array}$ & Conformer I & Conformer II & Conformer III & Conformer IV \\
\hline \multicolumn{5}{|c|}{ Bond lengths (pm) } \\
\hline$r\left(\mathrm{H}_{1}-\mathrm{Se}\right)$ & 145.2 & 145.3 & 145.3 & 145.3 \\
\hline$r\left(\mathrm{Se}-\mathrm{C}_{1}\right)$ & 195.7 & 194.7 & 195.1 & 195.4 \\
\hline$r\left(\mathrm{C}_{1}-\mathrm{H}_{2}\right)$ & 108.8 & 109.0 & 108.8 & 108.8 \\
\hline$r\left(\mathrm{C}_{1}-\mathrm{H}_{3}\right)$ & 108.8 & 108.8 & 109.1 & 108.9 \\
\hline$r\left(\mathrm{C}_{1}-\mathrm{C}_{2}\right)$ & 150.6 & 150.8 & 149.6 & 149.7 \\
\hline$r\left(\mathrm{C}_{2}-\mathrm{H}_{4}\right)$ & 108.3 & 108.4 & 108.2 & 108.2 \\
\hline$r\left(\mathrm{C}_{2}-\mathrm{C}_{3}\right)$ & 150.3 & 150.1 & 150.8 & 150.6 \\
\hline$r\left(\mathrm{C}_{2}-\mathrm{C}_{4}\right)$ & 150.3 & 150.2 & 149.9 & 150.4 \\
\hline$r\left(\mathrm{C}_{3}-\mathrm{H}_{5}\right)$ & 107.9 & 107.9 & 108.0 & 107.9 \\
\hline$r\left(\mathrm{C}_{3}-\mathrm{H}_{6}\right)$ & 108.0 & 108.1 & 108.1 & 108.0 \\
\hline$r\left(\mathrm{C}_{3}-\mathrm{C}_{4}\right)$ & 151.1 & 150.9 & 150.9 & 150.8 \\
\hline$r\left(\mathrm{C}_{4}-\mathrm{H}_{7}\right)$ & 107.9 & 107.9 & 107.9 & 107.9 \\
\hline$r\left(\mathrm{C}_{4}-\mathrm{H}_{8}\right)$ & 108.0 & 108.0 & 108.1 & 108.1 \\
\hline \multicolumn{5}{|c|}{ Bond angles (deg.) } \\
\hline$\angle\left(\mathrm{H}_{1}, \mathrm{Se}, \mathrm{C}_{1}\right)$ & 93.4 & 95.1 & 94.2 & 95.5 \\
\hline$\angle\left(\mathrm{Se}, \mathrm{C}_{1}, \mathrm{C}_{2}\right)$ & 111.9 & 115.9 & 113.3 & 108.0 \\
\hline$\angle\left(\mathrm{H}_{2}, \mathrm{C}_{1}, \mathrm{C}_{2}\right)$ & 110.4 & 110.4 & 112.3 & 111.7 \\
\hline$\angle\left(\mathrm{H}_{3}, \mathrm{C}_{1}, \mathrm{C}_{2}\right)$ & 110.4 & 111.3 & 110.4 & 110.5 \\
\hline$\angle\left(\mathrm{C}_{1}, \mathrm{C}_{2}, \mathrm{C}_{3}\right)$ & 122.2 & 121.8 & 118.5 & 118.8 \\
\hline$\angle\left(\mathrm{C}_{1}, \mathrm{C}_{2}, \mathrm{C}_{4}\right)$ & 122.2 & 122.4 & 118.8 & 118.4 \\
\hline$\angle\left(\mathrm{C}_{1}, \mathrm{C}_{2}, \mathrm{H}_{4}\right)$ & 112.5 & 112.6 & 115.4 & 115.4 \\
\hline$\angle\left(\mathrm{C}_{2}, \mathrm{C}_{3}, \mathrm{H}_{5}\right)$ & 117.8 & 118.0 & 118.2 & 117.9 \\
\hline$\angle\left(\mathrm{C}_{2}, \mathrm{C}_{3}, \mathrm{H}_{6}\right)$ & 117.5 & 116.8 & 117.0 & 117.2 \\
\hline$\angle\left(\mathrm{C}_{2}, \mathrm{C}_{4}, \mathrm{H}_{7}\right)$ & 117.8 & 118.0 & 118.3 & 118.3 \\
\hline$\angle\left(\mathrm{C}_{2}, \mathrm{C}_{4}, \mathrm{H}_{8}\right)$ & 117.5 & 117.2 & 116.6 & 116.5 \\
\hline
\end{tabular}


TABLE 11S (cont.): Geometries of Four Conformers of Cyclopropylmethylselenol from ab initio Geometry Optimizations at the MP2/aug-cc-pVTZ Level of Theory

\begin{tabular}{|c|c|c|c|c|}
\hline $\begin{array}{l}\text { Structural } \\
\text { parameter }\end{array}$ & Conformer I & Conformer II & Conformer III & Conformer \\
\hline \multicolumn{5}{|c|}{ Dihedral angles (deg.) } \\
\hline$\angle\left(\mathrm{H}_{1}, \mathrm{Se}, \mathrm{C}_{1}, \mathrm{C}_{2}\right)$ & 180.0 & 80.2 & 64.4 & 169.4 \\
\hline$\angle\left(\mathrm{Se}, \mathrm{C}_{1}, \mathrm{C}_{2}, \mathrm{C}_{3}\right)$ & 36.4 & 38.2 & 150.3 & 148.7 \\
\hline$\angle\left(\mathrm{Se}, \mathrm{C}_{1}, \mathrm{C}_{2}, \mathrm{C}_{4}\right)$ & -36.4 & -34.6 & 80.6 & 79.1 \\
\hline$\angle\left(\mathrm{Se}, \mathrm{C}_{1}, \mathrm{C}_{2}, \mathrm{H}_{4}\right)$ & 180.0 & -178.7 & -64.7 & -65.6 \\
\hline$\angle\left(\mathrm{H}_{1}, \mathrm{Se}, \mathrm{C}_{1}, \mathrm{H}_{2}\right)$ & -58.7 & -158.5 & -60.9 & 48.1 \\
\hline$\angle\left(\mathrm{H}_{1}, \mathrm{Se}, \mathrm{C}_{1}, \mathrm{H}_{3}\right)$ & 58.7 & -44.8 & -175.6 & -70.8 \\
\hline$\angle\left(\mathrm{C}_{1}, \mathrm{C}_{2}, \mathrm{C}_{3}, \mathrm{H}_{5}\right)$ & 140.6 & 139.8 & 143.6 & 144.1 \\
\hline$\angle\left(\mathrm{C}_{1}, \mathrm{C}_{2}, \mathrm{C}_{3}, \mathrm{H}_{6}\right)$ & -4.3 & -5.9 & -1.1 & -0.4 \\
\hline$\angle\left(\mathrm{C}_{1}, \mathrm{C}_{2}, \mathrm{C}_{4}, \mathrm{H}_{7}\right)$ & -140.6 & -140.8 & -143.9 & -143.5 \\
\hline$\angle\left(\mathrm{C}_{1}, \mathrm{C}_{2}, \mathrm{C}_{4}, \mathrm{H}_{8}\right)$ & 4.3 & 5.1 & 0.1 & 0.8 \\
\hline
\end{tabular}

